\title{
Derivation of Feynman rules for higher order poles using cross-ratio identities in $\mathrm{CHY}$ construction
}

\author{
Kang Zhou, ${ }^{a}$ Junjie Rao $^{b}$ and Bo Feng ${ }^{b, c}$ \\ ${ }^{a}$ School of Mathematical Sciences, Zhejiang University, \\ No. 38, Zheda Road, Hangzhou, 310027, P.R. China \\ ${ }^{b}$ Zhejiang Institute of Modern Physics, Department of Physics, Zhejiang University, \\ No. 38, Zheda Road, Hangzhou, 310027, P.R. China \\ ${ }^{c}$ Center of Mathematical Science, Zhejiang University, \\ No. 38, Zheda Road, Hangzhou, 310027, P.R. China \\ E-mail: zkzrzm@126.com, raojunjie@zju.edu.cn, fengbo@zju.edu.cn
}

ABSTRACT: In order to generalize the integration rules to general CHY integrands which include higher order poles, algorithms are proposed in two directions. One is to conjecture new rules, and the other is to use the cross-ratio identity method. In this paper,we use the cross-ratio identity approach to re-derive the conjectured integration rules involving higher order poles for several special cases: the single double pole, single triple pole and duplexdouble pole. The equivalence between the present formulas and the previously conjectured ones is discussed for the first two situations.

KEYwORDS: Gauge Symmetry, Scattering Amplitudes

ArXiv EPrint: 1705.04783 


\section{Contents}

1 Introduction 1

2 Preparation 2

3 Rule I: single double pole $\quad 4$

3.1 CHY configuration 5

3.2 Some examples 6

3.3 Analytic proof 8

4 Rule II: single triple pole $\quad 10$

$\begin{array}{lll}\text { 4.1 CHY configuration } & 10\end{array}$

4.2 Derivation of new Feynman rules 11

$\begin{array}{ll}4.3 & \text { Comparison with conjectured formula } \\ \end{array}$

5 Rule for duplex-double pole $\quad 21$

5.1 CHY configuration 21

5.2 Derivation 22

6 Conclusion 26

\section{Introduction}

The well-known Cachazo-He-Yuan (CHY) formulation [1-5] is an elegant new representation of tree-level amplitudes for massless particles in arbitrary dimensions, as given by

$$
\mathcal{A}_{n}=\int \frac{\prod_{i=1}^{n} d z_{i}}{\operatorname{vol~SL}(2, \mathbb{C})} \prod_{a}^{\prime} \delta\left(\mathcal{E}_{a}\right) I_{n}^{\mathrm{CHY}},
$$

which possesses the Möbius $\operatorname{SL}(2, \mathbb{C})$ invariance. It expresses amplitudes of a large variety of quantum field theories as multi-dimensional contour integrals over auxiliary variables $z_{i}$ 's, which are completely localized on the Riemann sphere by constraints known as the scattering equations

$$
\mathcal{E}_{a} \equiv \sum_{b \in\{1,2, \ldots, n\} \backslash\{a\}} \frac{s_{a b}}{z_{a b}}=0, \quad a=1,2, \ldots, n,
$$

where $s_{a b} \equiv 2 k_{a} \cdot k_{b}$ is the Mandelstam variable and $z_{a b}$ is defined as $z_{a b} \equiv z_{a}-z_{b}$. The scattering equations and the integration measure are universal, while the integrand $I_{n}^{\mathrm{CHY}}$ obeys some general constraints (such as of weight-4 under Möbius transformations), and it also depends on the specific field theory.

This formulation indicates that a tree amplitude can be calculated by solving scattering equations and summing over different solutions. However, it is hardly possible to get direct solutions beyond five points due to the Abel-Ruffini theorem for algebraic systems. Thus, to search for a new computation method to avoid the explicit solutions of algebraic equations 
becomes a crucial challenge. Among investigations from various directions [6-18], the socalled integration rule method inspired by string theory is one of the most efficient and systematic approaches [17-19]. This approach replies only on the CHY integrands, without mentioning the solutions of scattering equations and the integration measure. Applying this method, one can extract all the correct pole structures from a given integrand, and directly obtain the result via the corresponding Feynmann diagrams, rather than solving the scattering equations. However, one shortcomings of the original integration rules [17-19] is that it requires the $\mathrm{CHY}$ integrand under consideration containing simple poles only, therefore cannot be applied to arbitrary physically acceptable integrands in general.

To handle this disadvantage, there are two alternative approaches. One is to derive integration rules for higher order poles [20], and the other is to reduce terms containing higher order poles into those with simple poles only [17, 21]. In the first direction, integration rules for several special configurations of CHY integrands with higher order poles are conjectured in [20]. Although its analytic proof is absent, these rules are numerically verified. A more hopeful approach comes from the second direction, thanks to the discovery of the cross-ratio identities, which reveals relations between different rational functions of $z_{i j}$ 's [22]. By applying these identities iteratively, one can expand a term involving higher order poles as terms with simple poles only. After this decomposition, one can obtain the integrated result through the original integration rules. The fesasibility of this algorithm is verified in [26]. Further applications of the cross-ratio identities can be seen in [23-25].

It is natural to ask: can we prove the conjectured integration rules of higher order poles in [20] via the cross-ratio identity method? In this paper, we will derive these rules analytically by applying those identities. Our derivation depends on the choices of the cross-ratio identities, thus different choices yield different expressions for the same pole configuration. The expressions of rules in this paper will be different from those conjectured in [20], and we will prove their equivalence for two cases.

This paper is organized as follows. In section 2, we summarize some useful notations, and some general properties of the CHY integrands and cross-ratio identities. In section 3, we derive the integration rule for $\mathrm{CHY}$ integrands containing a single double pole. In section 4 , we derive the integration rule for that containing a single triple pole. For this case, the equivalence between our formula and the conjectured one is rather non-trivial, and the proof of their equivalence indicates a new kind of integration rule, as will be discussed explicitly. In section 5, we derive the integration rule for those containing duplex-double poles, regarding a simplest special case. A brief conclusion is given in section 6 .

\section{Preparation}

Before going to the details, for clarity we give a summary of notations. Each CHY integrand corresponds to a weight- 4 graph, in which $n$ nodes are connected by a number of lines. Each line corresponds to a factor $z_{i j} .{ }^{1}$ Furthermore, since factor $z_{i j}$ can appear in both the numerator and the denominator, to distinguish them, we use a solid line to represent $z_{i j}$ in the denominator and a dashed line to represent that in the numerator. With this

\footnotetext{
${ }^{1}$ To distinguish them from lines in Feynman diagrams, we will call the latter "Feynman lines".
} 
assignment, the weight- 4 condition becomes that there are four lines connecting to each node, where a solid line is counted as +1 and a dashed line -1 .

For a set $\Lambda$ containing $|\Lambda|$ points of $z_{i}$, we call a line connecting two points in $\Lambda$ the internal line of $\Lambda$, and a line connecting at least one point inside $\Lambda$ and the other outside $\Lambda$ the external line of $\Lambda$. The number of internal lines of $\Lambda$ is denoted by $\mathbb{L}[\Lambda]$, and that of external lines by $\mathbb{E}[\Lambda] .{ }^{2}$ Furthermore, we denote the number of lines connecting two sets $\Lambda_{1}$ and $\Lambda_{2}$ as $\mathbb{L}\left[\Lambda_{1}, \Lambda_{2}\right]$.

The order of poles corresponding to the set $\Lambda$ is defined as

$$
\chi[\Lambda]=\mathbb{L}[\Lambda]-2(|\Lambda|-1) .
$$

For convenience we call a set corresponding to simple poles as a "simple set", and similar for sets corresponding to double and triple poles. Due to the weight- 4 condition, we have $4|\Lambda|=2 \mathbb{L}[\Lambda]+\mathbb{E}[\Lambda]$, thus (2.1) can be rewritten as

$$
\chi[\Lambda]=2-\frac{\mathbb{E}[\Lambda]}{2},
$$

which will be useful later. Then, we have the following corollaries:

- A simple set has 4 external lines.

- A double set has 2 external lines.

- A triple set has 0 external lines.

- If a set $\Lambda$ contains only one point $i$, i.e., $|\Lambda|=1$, we have $\mathbb{E}[\Lambda]=4$ and thus $\chi[\Lambda]=0$ from (2.2). Although it does not contribute any simple pole, we still call it a simple set.

A set $\Lambda$ may contain many subsets which correspond to different poles. Similar to the definition of compatible combinations for the full set of $z_{i}$ 's, one can define the compatible combinations for the set $\Lambda$, and denote the sum of these combinations as $\mathcal{C}[\Lambda]$. The difference is, if the full set $\Lambda$ contributes a pole, $\mathcal{C}[\Lambda]$ must include this pole. For example, for a set $\{1,2,3,4\}$, if the full set does not correspond to any pole but $\{1,2,3\},\{1,2\},\{3,4\}$ contribute poles, then $\mathcal{C}[\Lambda]$ is defined $\mathrm{as}^{3}$

$$
\mathcal{C}[\Lambda]=\frac{1}{s_{123} s_{12}}+\frac{1}{s_{12} s_{34}} .
$$

However, if the full set contributes a pole, $\mathcal{C}[\Lambda]$ now should be

$$
\mathcal{C}[\Lambda]=\frac{1}{s_{1234}}\left(\frac{1}{s_{123} s_{12}}+\frac{1}{s_{12} s_{34}}\right) .
$$

For the set with a single point $i$, we have $\mathcal{C}[\{i\}]=1$.

\footnotetext{
${ }^{2}$ Again, the number of lines are counted as +1 for a solid line and -1 for a dashed line.

${ }^{3}$ When we write down (2.3) and (2.4), we have assumed that all poles are simple. For non-simple poles, further modification is needed.
} 
Each CHY integrand may give a number of terms and each term can be represented by a Feynman diagram with only cubic vertices. Except the special case of $n=3$ (i.e., there are only three external nodes), each cubic vertex contains at least one internal propagator. At each endpoint of this internal propagator (there are two), two branches are produced. They can be two external nodes, or one external node and one internal propagator, or two internal propagators. No matter in which situation, this internal propagator will be associated with a subset $\Lambda$ and its complement $\bar{\Lambda}$, thus we have

$$
\mathcal{C}[\Lambda]=\sum_{\left\langle\Lambda_{1} \Lambda_{2}\right\rangle} \frac{1}{s_{\Lambda}^{\chi+1}} \mathcal{C}\left[\Lambda_{1}\right] \mathcal{C}\left[\Lambda_{2}\right],
$$

where $\Lambda_{1}$ and $\Lambda_{2}$ are two branches (two subsets with $\Lambda_{1} \bigcup \Lambda_{2}=\Lambda$ ) associated with the endpoints of subset $\Lambda$. The summation is over all correct divisions of $\Lambda_{1}, \Lambda_{2}$, and a special division is denoted as $\left\langle\Lambda_{1} \Lambda_{2}\right\rangle$.

The major machinery we use in this paper is the cross ratio identity

$$
-s_{\Lambda}=-s_{\bar{\Lambda}}=\sum_{i \in \Lambda /\{p\}} \sum_{j \in \bar{\Lambda} /\{q\}} s_{i j} \frac{z_{i p} z_{j q}}{z_{i j} z_{p q}}
$$

given in [22]. Let us give some explanations of (2.6):

(1) $(p, q)$ is the gauge choice. Although different gauge choices give equivalent expressions, some choices will simplify the calculation.

(2) We have double sums over all subsets $\Lambda$ and $\bar{\Lambda}$.

(3) For each term in the sum, we have a kinematic factor $s_{i j}$. The two denominators $z_{i j}$ and $z_{p q}$ (fixed for all terms in the sum) between subsets $\Lambda$ and $\bar{\Lambda}$ increase $\mathbb{E}[\Lambda]$ and $\mathbb{E}[\bar{\Lambda}]$ by 2 , thus from $(2.2), \chi[\Lambda]$ and $\chi[\bar{\Lambda}]$ are reduced by 1 . Similarly, two numerators, i.e., $z_{i p}$ in the subset $\Lambda$ and $z_{j q}$ in $\bar{\Lambda}$, reduce $\mathbb{L}[\Lambda]$ and $\mathbb{L}[\bar{\Lambda}]$ by 1 , thus from $(2.1), \chi[\Lambda]$ and $\chi[\bar{\Lambda}]$ are reduced by 1 .

As when applying the cross-ratio identities, one needs to choose a gauge which includes two points and a set corresponding to a pole, for simplicity we use $[p, q, \Lambda]$ to denote the gauge choice, as well as the corresponding pole.

\section{Rule I: single double pole}

In this section, we will derive the Feynman rule I for a single double pole. The corresponding conjectured formula in [20] is given as

$$
\mathcal{R}_{\text {ule }}^{\mathrm{I}}\left[p_{A}, p_{B}, p_{C}, p_{D}\right]=\frac{2 p_{A} \cdot p_{C}+2 p_{B} \cdot p_{D}}{2 s_{A B}^{2}} .
$$




\subsection{CHY configuration}

First we try to understand CHY configurations with only one double pole $s_{\Lambda}$. This means that all subsets $A$ have $\chi[A] \leq 0$, except one subset $\Lambda$ with $\chi[\Lambda]=1$. Furthermore, for simplicity we will assume the numerator of CHY integrand is just $1 .{ }^{4}$ With the assumption above, we can give some statements of CHY configurations.

First, we have

$$
\mathbb{E}[\Lambda]=2,
$$

which means that there are two and only two lines connecting subset $\Lambda$ and its complement $\bar{\Lambda}$. Now we will show that these two lines cannot meet at the same node. Let us assume the two lines are given by a point $a \in \Lambda$ connecting to $\bar{\Lambda}$, then

$$
\begin{aligned}
\chi[\Lambda /\{a\}] & =\mathbb{L}[\Lambda /\{a\}]-2(|\Lambda|-1-1)=\mathbb{L}[\Lambda]-2-2(|\Lambda|-1-1) \\
& =\mathbb{L}[\Lambda]-2(|\Lambda|-1)=1,
\end{aligned}
$$

where we have used the fact that point $a$ has four connecting lines, so there are two lines connecting point $a$ and the subset $\Lambda /\{a\}$. Since we have assumed that all $\chi[A] \leq 0$ with $A \neq \Lambda$, (3.3) contradicts with this assumption. This means that there are two points $a_{1}, a_{2} \in \Lambda$ and two points $b_{1}, b_{2} \in \bar{\Lambda}$, such that there are one line connecting $a_{1}, b_{1}$ and the other connecting $a_{2}, b_{2}$. As will be discussed cautiously, when we apply the cross-ratio identities to find the Feynman rule, the good gauge choice is either $\left[a_{1}, b_{2}, \Lambda\right]$ or $\left[a_{2}, b_{1}, \Lambda\right]{ }^{5}$

Second, we will show that there is no subset $A \subset \Lambda$ containing both $a_{1}, a_{2}$ satisfying $\chi[A]=0$. If such a subset exists, we can consider its complement $\widetilde{A}=\Lambda / A$. Since $\chi[A]=0$, we have $\mathbb{E}[A]=4$. Because $a_{1}, a_{2} \in A$, we have $\mathbb{L}[A, \widetilde{A}]=2$. Now since $a_{1}, a_{2} \notin \widetilde{A}$, we have $\mathbb{E}[\widetilde{A}]=2$ so $\chi[\widetilde{A}]=1$, which contradicts with our assumption. Thus, four nodes $a_{0}, b_{0}, c_{0}$ and $d_{0}$ belong to four different simple subsets, as shown in figure 1a.

Third, we will show that there is no subset $\Sigma=\alpha \bigcup \beta$, such that $\alpha \subset \Lambda, \beta \subset \bar{\Lambda}$ satisfying $\chi[\Sigma]=0$. If such a subset exists, we have

$$
\begin{aligned}
0 & =\chi[\Sigma]=\mathbb{L}[\Sigma]-2(|\Sigma|-1)=\mathbb{L}[\alpha]+\mathbb{L}[\beta]+\mathbb{L}[\alpha, \beta]-2(|\alpha|+|\beta|-1) \\
& =(\mathbb{L}[\alpha]-2(|\alpha|-1))+(\mathbb{L}[\beta]-2(|\beta|-1))+(\mathbb{L}[\alpha, \beta]-2) .
\end{aligned}
$$

Since from our assumption $\chi[\alpha] \leq 0$ and $\chi[\beta] \leq 0$, we have $(\mathbb{L}[\alpha, \beta]-2) \geq 0$. With the condition $\mathbb{L}[\alpha, \beta] \leq \mathbb{L}[\Lambda, \bar{\Lambda}]=2$, the equation above holds when and only when

$$
\chi[\alpha]=0, \quad \chi[\beta]=0, \quad \mathbb{L}[\alpha, \beta]=2 \Longrightarrow a_{1}, a_{2} \in \alpha, \quad b_{1}, b_{2} \in \beta,
$$

which contradicts with the second observation in the previous paragraph.

\footnotetext{
${ }^{4}$ Although we will not give a rigorous proof for CHY integrands with nontrivial numerators in this paper, we believe Feynman rule I will be applicable to this more general situation. In fact, when we derive the rule III, we will meet the situation where although the numerator is not one, the same rule I has been applied to get the correct results.

${ }^{5}$ As we have remarked, the claim above, i.e., there are only two lines $z_{a_{1} b_{1}}$ and $z_{a_{2} b_{2}}$, has neglected the possibility that there are nontrivial numerators in CHY integrands, which will bring more solid lines (i.e., factors in the denominator) connecting $\Lambda, \bar{\Lambda}$. To deal with this case, one can use, for example,

$$
\frac{z_{a b} z_{d c}}{z_{a c} z_{b c}}=\frac{z_{a d}}{z_{a c}}-\frac{z_{b d}}{z_{b c}}
$$

to get rid of the numerator. In this paper, for simplicity we will not discuss such more general configurations.
} 


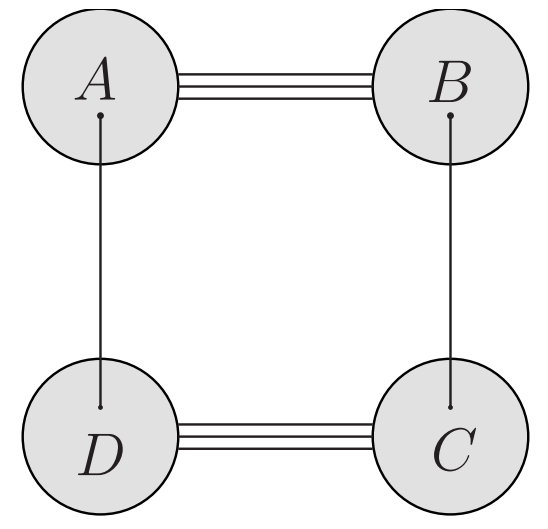

(a) Original integrand

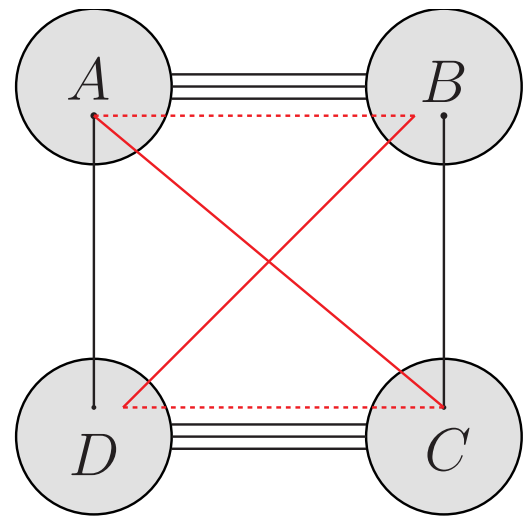

(b) Cross-ratio identity

Figure 1. Configuration of the original integrand and the cross-ratio identity of the rule I. Four red lines represent the term $\frac{z_{i a_{0}} z_{j c_{0}}}{z_{i j} z_{a_{0} c_{0}}}$ provided by the cross-ratio identity.

With these observations for single pole structures, we move to the maximal compatible combinations of poles, i.e., the possible Feynman diagrams. The claim is: all maximal compatible combinations must contain the double subset $\Lambda$. The reason is: to get nonzero contributions, the number of compatible subsets must be $n-3$. Since we have argued that there is no single pole $\Sigma=\alpha \bigcup \beta$ with $\alpha \subset \Lambda, \beta \subset \bar{\Lambda}$, all single poles are either in $\Lambda$ or in $\bar{\Lambda}$. But $\Lambda$ can contain at most $(|\Lambda|-2)$ compatible poles, while $\bar{\Lambda}$ can contain at most $(|\bar{\Lambda}|-2)$ ones. Thus their combinations contribute only $(n-4)$ compatible single poles, and we need to include the double pole to get nonzero contributions.

\subsection{Some examples}

To demonstrate how to derive the Feynman rule using cross ratio identities, we present several examples in this subsection. The same picture will be used when derive other Feynman rules later although explicit examples will not be given.

Let us start with the simplest example with only four points with the CHY integrand

$$
I_{4 ; a}=\frac{1}{z_{12}^{3} z_{34}^{3} z_{23} z_{41}} .
$$

This example contains only one double pole $s_{\Lambda}$ with $\Lambda=\{1,2\}$. There are two lines [14], [23] connecting $\Lambda$ and $\bar{\Lambda}=\{3,4\}$. Now we apply the cross ratio identities. There are two different gauge choices, for the first one $[2,3, \Lambda]$, we have

$$
I_{4 ; a}=\frac{1}{z_{12}^{3} z_{34}^{3} z_{23} z_{41}}\left(\frac{-1}{s_{12}} s_{14} \frac{z_{43} z_{12}}{z_{14} z_{23}}\right)=\frac{-s_{14}}{s_{12}} \frac{1}{z_{12}^{2} z_{34}^{2} z_{23}^{2} z_{41}^{2}}=\frac{-s_{14}}{s_{12}}\left(\frac{-1}{s_{12}}+\frac{-1}{s_{23}}\right)=\frac{-s_{13}}{s_{12}^{2}} .
$$

For the second one $[2,4, \Lambda]$, we have

$$
I_{4 ; a}=\frac{1}{z_{12}^{3} z_{34}^{3} z_{23} z_{41}}\left(\frac{-1}{s_{12}} s_{13} \frac{z_{34} z_{12}}{z_{13} z_{24}}\right)=\frac{-s_{13}}{s_{12}} \frac{1}{z_{12}^{2} z_{34}^{2} z_{23} z_{41} z_{13} z_{24}}=\frac{-s_{13}}{s_{12}} \frac{1}{s_{12}}=\frac{-s_{13}}{s_{12}^{2}} .
$$


We see that for the first gauge choice, pole $s_{23}$ is introduced since in addition to original lines $z_{12}, z_{34}$, new lines $z_{23}, z_{14}$ have been added by the cross ratio identity. For the second gauge choice, no new pole is introduced, so its calculation is simpler.

This phenomenon in fact suggests the general pattern. From the discussion in the previous subsection, for a given Feynman diagram, $\Lambda$ has been split to two subsets $A_{1}, A_{2}$ and $\bar{\Lambda}$ has been split to two subsets $B_{1}, B_{2}$ such that $a_{i} \in A_{i}, b_{i} \in B_{i}, i=1,2$. If the gauge choice is $\left[p \in A_{2}, q \in B_{2}, \Lambda\right]$, it is possible to find two subsets $\alpha \subset A_{2}, \beta \subset B_{2}$ satisfying the following conditions

$$
\chi[\alpha]=0, \quad \chi[\beta]=0, \quad a_{2} \in \alpha, p \notin \alpha ; \quad b_{2} \in \beta, q \notin \beta \Longrightarrow \mathbb{L}[\alpha, \beta]=1 .
$$

Thus after multiplying the CHY integrand by $s_{i j} \frac{z_{i p} z_{j q}}{z_{i j} z_{p q}}$, we have

$$
\chi[\alpha \bigcup \beta]=\mathbb{L}[\alpha]+\mathbb{L}[\beta]+\mathbb{L}[\alpha, \beta]+1-2(|\alpha|+|\beta|-1)=0,
$$

where the extra +1 comes from the denominator $z_{i j}$ for any $i \in \alpha, j \in \beta$. In other words, we will get a new pole $s_{\alpha \cup \beta}$, which does not exist in the original integrand. If we adopt the gauge $p \in A_{2}, q \in B_{1}$, such a phenomenon will not happen since $\mathbb{L}\left[A_{2}, B_{1}\right]=0$. Thus to avoid this problem, from now, we will always adopt the gauge $p \in A_{2}, q \in B_{1}$ or $p \in A_{1}, q \in B_{2}$. Furthermore, since the splitting of $\Lambda$ into $A_{1}, A_{2}$ is, in general, arbitrary, it is hard to guarantee that a node $a$ always stays in $A_{1}$. The only exception is that nodes $a_{1}, a_{2}$ (which connect to $b_{1}, b_{2} \in \bar{\Lambda}$ ) from the second observation in the previous subsection, i.e., $a_{1}$ and $a_{2}$ are always in different subsets of the split $\Lambda$. Thus there is a universal gauge choice for all Feynman diagrams, which can be either $\left[a_{1}, b_{2}, \Lambda\right]$ or $\left[a_{2}, b_{1}, \Lambda\right] .{ }^{6}$

The next example is the 5-point CHY integrand

$$
I_{5 ; a}=-\frac{1}{z_{12}^{3} z_{23} z_{34}^{2} z_{45}^{2} z_{53} z_{51}}
$$

which, according to the Feynman rule (3.1), will lead to

$$
\begin{aligned}
& \frac{1}{s_{34}} \mathcal{R}_{\text {ule }}^{\mathrm{I}}[\{1\},\{2\},\{3,4\},\{5\}]+\frac{1}{s_{45}} \mathcal{R}_{\text {ule }}^{\mathrm{I}}[\{1\},\{2\},\{3\},\{4,5\}] \\
& \quad=\frac{1}{s_{34}} \frac{2 p_{1} p_{34}+2 p_{2} p_{5}}{2 s_{12}^{2}}+\frac{1}{s_{45}} \frac{2 p_{1} p_{3}+2 p_{2} p_{45}}{2 s_{12}^{2}}=\frac{s_{25}}{s_{34} s_{12}^{2}}+\frac{s_{13}}{s_{45} s_{12}^{2}}-\frac{1}{s_{12}^{2}} .
\end{aligned}
$$

Now we derive this result using the cross ratio identity for the double pole $s_{12}$ (so $\Lambda=\{1,2\}$ and $\bar{\Lambda}=\{3,4,5\}$. The two lines connecting $\Lambda, \bar{\Lambda}$ are $z_{15}$ and $z_{23}$. Under the gauge $[2,5, \Lambda],{ }^{7}$

\footnotetext{
${ }^{6}$ As it will be seen in the derivation of Feynman rule III, in general we can not find the good gauge choice such that there is no extra pole introduced. At the same place, we will show how these extra poles have been canceled when summing all contributions together.

${ }^{7}$ If we adopt the gauge $[2,3, \Lambda]$, it will produce an extra pole $s_{23}$. If we adopt $[2,4, \Lambda]$, it will produce an extra pole $s_{15}$. Thus, to avoid new poles, in this gauge choice 5 is the only option if we have chosen 2 .
} 
we have

$$
\begin{aligned}
- & \frac{1}{z_{12}^{3} z_{23} z_{34}^{2} z_{45}^{2} z_{53} z_{51}} \frac{-1}{s_{12}}\left(s_{13} \frac{z_{12} z_{35}}{z_{13} z_{25}}+s_{14} \frac{z_{12} z_{45}}{z_{14} z_{25}}\right) \\
& =\frac{s_{13}}{s_{12}} \frac{z_{35}}{z_{12}^{2} z_{23} z_{34}^{2} z_{45}^{2} z_{53} z_{51} z_{13} z_{25}}+\frac{s_{14}}{s_{12}} \frac{z_{45}}{z_{12}^{2} z_{23} z_{34}^{2} z_{45}^{2} z_{53} z_{51} z_{14} z_{25}} \\
& =\frac{s_{13}}{s_{12}}\left(\frac{1}{s_{12} s_{34}}+\frac{1}{s_{12} s_{45}}\right)+\frac{s_{14}}{s_{12}}\left(\frac{1}{s_{12} s_{34}}\right) \\
& =\frac{s_{13}+s_{14}}{s_{12}^{2} s_{34}}+\frac{s_{13}}{s_{12}^{2} s_{45}}=\frac{2 p_{1} \cdot p_{34}}{s_{12}^{2} s_{34}}+\frac{2 p_{1} \cdot p_{3}}{s_{12}^{2} s_{45}} .
\end{aligned}
$$

It seems that we get only a part of the result (3.13). Under the gauge $[1,3, \Lambda]$ we have

$$
\begin{aligned}
& - \\
& -\frac{1}{z_{12}^{3} z_{23} z_{34}^{2} z_{45}^{2} z_{53} z_{51}} \frac{-1}{s_{12}}\left(s_{25} \frac{z_{21} z_{53}}{z_{13} z_{25}}+s_{24} \frac{z_{21} z_{43}}{z_{24} z_{13}}\right) \\
& \quad=\frac{s_{25}}{s_{12}} \frac{z_{35}}{z_{12}^{2} z_{23} z_{34}^{2} z_{45}^{2} z_{53} z_{51} z_{13} z_{25}}+\frac{s_{24}}{s_{12}} \frac{z_{34}}{z_{12}^{2} z_{23} z_{34}^{2} z_{45}^{2} z_{53} z_{51} z_{24} z_{13}} \\
& \quad=\frac{s_{25}}{s_{12}}\left(\frac{1}{s_{12} s_{34}}+\frac{1}{s_{12} s_{45}}\right)+\frac{s_{24}}{s_{12}}\left(\frac{1}{s_{12} s_{45}}\right)=\frac{2 p_{2} \cdot p_{5}}{s_{12}^{2} s_{34}}+\frac{2 p_{2} \cdot p_{45}}{s_{12}^{2} s_{45}} .
\end{aligned}
$$

Again this is not the full result but the other part of the result (3.13). Now we see the solution: summing up (3.14) and (3.15), we arrive

$$
\frac{s_{13}+s_{14}+s_{25}}{2 s_{12}^{2} s_{34}}+\frac{s_{13}+s_{25}+s_{24}}{2 s_{12}^{2} s_{45}}=\frac{2 p_{1} \cdot p_{34}+2 p_{2} \cdot p_{5}}{2 s_{12}^{2} s_{34}}+\frac{2 p_{1} \cdot p_{3}+2 p_{2} \cdot p_{45}}{2 s_{12}^{2} s_{45}},
$$

which matches the Feynman rule (3.13).

Although simple, this example reveals that: (1) It seems that we can define different "Feynman rules"; (2) Different Feynman rules come from different gauge choices.

One can use more examples to better understand these two observations, as they persist to all configurations of the current category. In the next subsection, we will give an analytic proof.

\subsection{Analytic proof}

Having understood those examples, now we can give a general analytic proof. First, from the assumption of CHY integrands, i.e., there is one and only one subset satisfying $\chi[\Lambda]=1$ and for all others $\chi[A] \leq 0$, we have the following statement:

- All maximal compatible combinations contain the subset $\Lambda$, i.e., all nonzero Feynman diagrams contain the double pole $\frac{1}{s_{\Lambda}^{2}}$.

- There are four special nodes $a_{0}, b_{0} \in \Lambda$ and $c_{0}, d_{0} \in \bar{\Lambda}$, such that there is one line connecting nodes $a_{0}, d_{0}$ and one connecting nodes $b_{0}, c_{0} \cdot{ }^{8}$

\footnotetext{
${ }^{8}$ Again, this claim holds only if we assume the numerator is just 1.
} 
Now we consider the Feynman rule with different gauge choices. For $\left[a_{0}, c_{0}, \Lambda\right]$, the corresponding cross ratio identity is

$$
1=\frac{-1}{s_{\Lambda}} \sum_{i \in \Lambda /\left\{a_{0}\right\}} \sum_{j \in \bar{\Lambda} /\left\{c_{0}\right\}} s_{i j} \frac{z_{i a_{0}} z_{j c_{0}}}{z_{i j} z_{a_{0} c_{0}}},
$$

as shown in figure 1b. Now we consider new CHY integrands $\mathcal{I}_{\operatorname{org}} \frac{z_{i a_{0}} z_{j c_{0}}}{z_{i j} z_{a_{0} c_{0}}}$ for each $(i, j)$ pair. First, all integrands contain only simple poles in our construction. Second, as we have argued, under this gauge choice, all possible poles are those already appeared in the original $\mathcal{I}_{\text {org }}$ and no new pole will appear. Based on these two facts, now we focus on the contributions to a particular Feynman diagram with the pole structure $\frac{1}{s_{A} s_{B} s_{C} s_{D}}$ (where $A \bigcup B=\Lambda$ and $C \bigcup D=\bar{\Lambda}, a_{0} \in A, b_{0} \in B, c_{0} \in C$ and $\left.d_{0} \in D\right)^{9}$ from these CHY integrands. For this pole structure, summation after inserting the cross ratio identity can be divided into the following four parts:

$$
\begin{aligned}
G\left(a_{0}, c_{0}\right)_{\mathrm{I}} & =\frac{-1}{s_{\Lambda}} \sum_{i \in A /\left\{a_{0}\right\}} \sum_{j \in C /\left\{c_{0}\right\}} s_{i j} \frac{z_{i a_{0}} z_{j c_{0}}}{z_{i j} z_{a_{0} c_{0}}} \mathcal{I}_{\mathrm{org}}, \\
G\left(a_{0}, c_{0}\right)_{\mathrm{II}} & =\frac{-1}{s_{\Lambda}} \sum_{i \in A /\left\{a_{0}\right\}} \sum_{j \in D} s_{i j} \frac{z_{i a_{0}} z_{j c_{0}}}{z_{i j} z_{a_{0} c_{0}}} \mathcal{I}_{\mathrm{org}}, \\
G\left(a_{0}, c_{0}\right)_{\mathrm{III}} & =\frac{-1}{s_{\Lambda}} \sum_{i \in B} \sum_{j \in C /\left\{c_{0}\right\}} s_{i j} \frac{z_{i a_{0}} z_{j c_{0}}}{z_{i j} z_{a_{0} c_{0}}} \mathcal{I}_{\mathrm{org}}, \\
G\left(a_{0}, c_{0}\right)_{\mathrm{IV}} & =\frac{-1}{s_{\Lambda}} \sum_{i \in B} \sum_{j \in D} s_{i j} \frac{z_{i a_{0}} z_{j c_{0}}}{z_{i j} z_{a_{0} c_{0}}} \mathcal{I}_{\text {org }} .
\end{aligned}
$$

Let us analyze them one by one. For $G\left(a_{0}, c_{0}\right)_{I}$, since $\chi(A)=0$ for the original integrand, after multiplying it by $\frac{z_{i a_{0}} z_{j c_{0}}}{z_{i j} z_{a_{0} c_{0}}}$, we have $\chi(A)=-1$ due to the numerator $z_{i a_{0}}$. In other words, $\mathcal{I}_{\text {org }} \frac{z_{i a_{0}} z_{j c_{0}}}{z_{i j} z_{a_{0} c_{0}}}$ will not contain the pole $\frac{1}{s_{A}}$. Similarly, the numerator $z_{j c_{0}}$ will lead to the fact that there is no pole $\frac{1}{s_{C}}$. Altogether, we find the $G\left(a_{0}, c_{0}\right)_{I}$ part will not contribute to the pole structure $\frac{1}{s_{A} s_{B} s_{C} s_{D}}$. The same argument tells that, we can exclude the contribution from $G\left(a_{0}, c_{0}\right)_{I I}$ and $G\left(a_{0}, c_{0}\right)_{I I I}$. For the $G\left(a_{0}, c_{0}\right)_{I V}$ part, each term gives the same contribution ${ }^{10}$ to the particular pole structure, and we find the total coefficient is given by

$$
\frac{-1}{s_{A B}} \sum_{i \in B} \sum_{j \in D} s_{i j}=\frac{2 p_{B} \cdot p_{D}}{s_{A B}} .
$$

Summing over all possible splittings $A, B, C, D$, we get

$$
\begin{aligned}
& I^{\mathrm{CHY}} \rightarrow \sum_{B \subset \Lambda} \sum_{D \subset \bar{\Lambda}} \frac{2 p_{B} \cdot p_{D}}{s_{\Lambda}^{2}} \mathcal{C}[A] \mathcal{C}[B] \mathcal{C}[C] \mathcal{C}[D], \\
& a_{0} \in A=\Lambda / B, b_{0} \in B, c_{0} \in C=\bar{\Lambda} / D, d_{0} \in D,
\end{aligned}
$$

\footnotetext{
${ }^{9}$ As we have proven, four special nodes must be in four different corners.

${ }^{10}$ It is easy to see that each term contributes the same pole structure, but it is hard to see that each term gives with same sign. The sign can, in principle, be determined by using either the method in [3] or that in [22]. The discussion of sign is too complicated for us to give a general, simple argument.
} 
which is one possible Feynman rule. From the same argument, one can see that for the gauge choice $\left[b_{0}, d_{0}, \Lambda\right]$, among four parts, only one gives nonzero contribution with the coefficient

$$
\frac{-1}{s_{A B}} \sum_{i \in A} \sum_{j \in C} s_{i j}=\frac{2 p_{A} \cdot p_{C}}{s_{A B}},
$$

thus the other possible Feynman rule is

$$
\begin{aligned}
& I^{\mathrm{CHY}} \rightarrow \sum_{A \subset \Lambda} \sum_{C \subset \bar{\Lambda}} \frac{2 p_{A} \cdot p_{C}}{s_{\Lambda}^{2}} \mathcal{C}[A] \mathcal{C}[B] \mathcal{C}[C] \mathcal{C}[D] \\
& a_{0} \in A, \quad b_{0} \in B=\Lambda / A, c_{0} \in C, d_{0} \in D=\bar{\Lambda} / C .
\end{aligned}
$$

Averaging over these two contributions, we get the Feynman rule

$$
\begin{gathered}
I^{\mathrm{CHY}} \rightarrow \sum_{A \subset \Lambda} \sum_{C \subset \bar{\Lambda}} \frac{2 p_{A} \cdot p_{C}+2 p_{B} \cdot p_{D}}{2 s_{\Lambda}^{2}} \mathcal{C}[A] \mathcal{C}[B] \mathcal{C}[C] \mathcal{C}[D], \\
a_{0} \in A, \quad b_{0} \in B=\Lambda / A, c_{0} \in C, d_{0} \in D=\bar{\Lambda} / C,
\end{gathered}
$$

which is the original Feynman rule we conjectured. We would like to emphasize that all three rules (3.20), (3.22) and (3.23) are correct, but when applying these rules, one must stick to the same rule for all Feynman diagrams (i.e., all possible splittings of subsets $\Lambda \rightarrow A \bigcup B$ and $\bar{\Lambda} \rightarrow C \bigcup D)$ in order to get the correct final answer.

\section{Rule II: single triple pole}

In this section, we consider CHY configurations with only one triple pole $s_{\Lambda}$. The equivalence between our formula and the conjectured one proposed in [20] is rather subtle, and it indicates a new kind of integration rule involving quartic vertices, as will be discussed cautiously.

\subsection{CHY configuration}

The assumption of only single triple pole requires that all subsets $A$ have $\chi[A] \leq 0$, except one subset $\Lambda$ with $\chi[\Lambda]=2$. Furthermore, for simplicity, we will assume the numerator of CHY integrand is just $1 .{ }^{11}$ With above assumption, we can make some statements of CHY configurations.

First, the triple set $\Lambda$ satisfies $\mathbb{E}[\Lambda]=0$, thus there is no line between $\Lambda$ and its complement subset $\bar{\Lambda}$. In other words, subset $\Lambda$ and $\bar{\Lambda}$ give weight- 4 graphes by themselves (or a legitimate $\mathrm{CHY}$ integrands for smaller nodes). As a consequence, if a subset $A \subset \Lambda$ gives a simple pole, so is $B=\Lambda \backslash A$ since $\mathbb{E}[A]=\mathbb{E}[B]=\mathbb{L}[A, B]=4$. Similarly, if subset $C \subset \bar{\Lambda}$ gives a simple pole, so is $D=\bar{\Lambda} / C$. To make things simpler, we will assume node $1 \in \Lambda$ and node $n \in \bar{\Lambda}$. Furthermore, when split at the two ending points of internal propagator $s_{\Lambda}$, we will assume that $1 \in A, n \in D$ and denote the splitting as $\langle A B C D\rangle$ with $B=\Lambda / A$ and $C=\bar{\Lambda} / D$. The corresponding configuration is shown in figure 2a.

\footnotetext{
${ }^{11}$ Again, although we do not have the proof, we think the Feynman rule could be applied to the case with nontrivial numerators.
} 


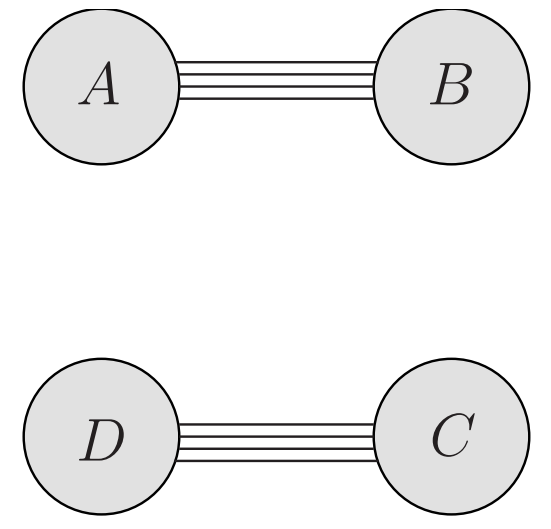

(a) Original integrand

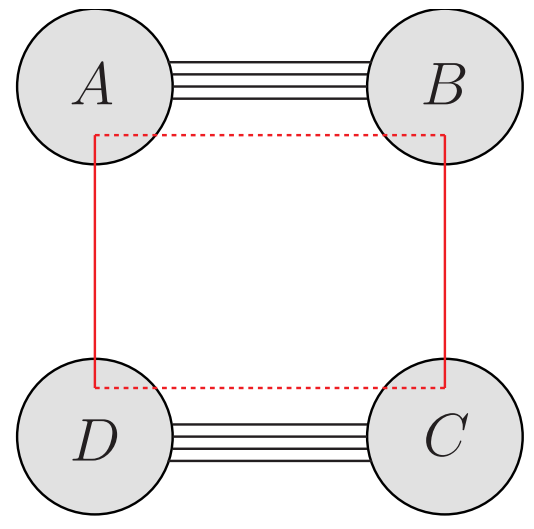

(b) Cross-ratio identity

Figure 2. Configuration of the original integrand and the cross-ratio identity of the rule II. Four red lines represent the term $\frac{z_{j n} z_{1 i}}{z_{i j} z_{1 n}}$ provided by the cross-ratio identity.

Secondly, using (3.5), one can observe that there is no pole corresponds to the set $\Sigma=\alpha \bigcup \beta$ such that $\alpha \subset \Lambda$ and $\beta \subset \bar{\Lambda}$, since $\chi_{\alpha} \leq 0, \chi_{\beta} \leq 0$ and $\mathbb{L}[\alpha, \beta]=0$. Finally, for any maximal compatible combination, by the same argument as in the previous section, one can see that it must contain the subset $\Lambda$. Thus, the CHY integrand will give the contribution like

$$
\sum_{i \in A \subset \Lambda} \sum_{n \in D \subset \bar{\Lambda}} \frac{X}{s_{\Lambda}^{3}} \mathcal{C}[A] \mathcal{C}[B] \mathcal{C}[C] \mathcal{C}[D],
$$

where the factor $X$ is what we need to derive for the Feynman rule.

\subsection{Derivation of new Feynman rules}

Having understood the configurations, now we drive the rule. Since it is the triple pole, we need to use the cross-ratio identities twice to reach simple poles. At the first step, we chose the gauge $[1, n, \Lambda]$ to get

$$
1=-\sum_{i \in \Lambda \backslash\{1\}} \sum_{j \in \bar{\Lambda} \backslash\{n\}} \frac{s_{i j}}{s_{\Lambda}} \frac{z_{j n} z_{i 1}}{z_{i j} z_{1 n}}=\sum_{i \in \Lambda \backslash\{1\}} \sum_{j \in \bar{\Lambda} \backslash\{n\}} \frac{s_{i j}}{s_{\Lambda}} \frac{z_{j n} z_{1 i}}{z_{i j} z_{1 n}},
$$

as can be seen in figure $2 \mathrm{~b}$. For given $(i, j)$, the CHY integrand $I_{\operatorname{org}} \frac{z_{j n} z_{1 i}}{z_{i j} z_{1 n}}$ is nothing but the configuration we have considered in previous section, i.e., the one with only one double pole. It is easy to see the point: the denominators $z_{i j}$ and $z_{1 n}$ have created two connecting lines between $\Lambda$ and $\bar{\Lambda}$. One may worry about the numerator $z_{j n}$ in $\Lambda$ and $z_{1 i}$ in $\bar{\Lambda}$. But as we have remarked, although our proof is given for the case with numerator 1 , we believe the rule holds for nontrivial numerator as current situation. Furthermore, at least for the current CHY graph, there is always a closed Hamiltonian circle containing 1 and $i$ in the subset $\Lambda$ (or $n$ and $j$ in the subset $\bar{\Lambda}$ ). Thus, one can use the open-relation in [23] (eq. (3.4)) or [25] (eq. (A12)) to eliminate the numerator (dashed line) to the case with numerator 1. 
Since the problem has been reduced to the case with only one double pole, we can use the previous result to write down

$$
\begin{aligned}
& I_{\mathrm{org}} \frac{z_{j n} z_{1 i}}{z_{i j} z_{1 n}} \rightarrow \sum_{B \subset \Lambda} \sum_{D \subset \bar{\Lambda}} \frac{2 p_{B} \cdot p_{D}}{s_{\Lambda}^{2}} \mathcal{C}[A] \mathcal{C}[B] \mathcal{C}[C] \mathcal{C}[D], \\
& 1 \in A=\Lambda / B, i \in B, j \in C=\bar{\Lambda} / D, n \in D
\end{aligned}
$$

for the gauge choice $[1, j, \Lambda]$ for the second step. Or

$$
\begin{aligned}
& I_{\mathrm{org}} \frac{z_{j n} z_{1 i}}{z_{i j} z_{1 n}} \rightarrow \sum_{A \subset \Lambda} \sum_{C \subset \bar{\Lambda}} \frac{2 p_{A} \cdot p_{C}}{s_{\Lambda}^{2}} \mathcal{C}[A] \mathcal{C}[B] \mathcal{C}[C] \mathcal{C}[D], \\
& 1 \in A, i \in B=\Lambda / A, j \in C, n \in D=\bar{\Lambda} / C,
\end{aligned}
$$

for the gauge choice $[i, n, \Lambda]$ for the second step. Now putting (4.3) and (4.4) back to (4.2) we get

$$
\begin{aligned}
& \sum_{i \in \Lambda \backslash\{1\}} \sum_{j \in \bar{\Lambda} \backslash\{n\}} \frac{s_{i j}}{s_{\Lambda}} \sum_{B \subset \Lambda} \sum_{D \subset \bar{\Lambda}} \frac{2 p_{B} \cdot p_{D}}{s_{\Lambda}^{2}} \mathcal{C}[A] \mathcal{C}[B] \mathcal{C}[C] \mathcal{C}[D], \\
& 1 \in A=\Lambda / B, i \in B, j \in C=\bar{\Lambda} / D, n \in D
\end{aligned}
$$

for the gauge choice $[1, j, \Lambda]$. Or

$$
\begin{aligned}
& \sum_{i \in \Lambda \backslash\{1\}} \sum_{j \in \bar{\Lambda} \backslash\{n\}} \frac{s_{i j}}{s_{\Lambda}} \sum_{A \subset \Lambda} \sum_{C \subset \bar{\Lambda}} \frac{2 p_{A} \cdot p_{C}}{s_{\Lambda}^{2}} \mathcal{C}[A] \mathcal{C}[B] \mathcal{C}[C] \mathcal{C}[D], \\
& 1 \in A, i \in B=\Lambda / A, j \in C, n \in D=\bar{\Lambda} / C,
\end{aligned}
$$

for the gauge choice $[i, n, \Lambda]$. To continue, we exchanging the ordering of summing to arrive at

$$
\begin{aligned}
& I_{\text {org }} \rightarrow \sum_{A \subset \Lambda} \sum_{D \subset \bar{\Lambda}} \frac{\left(2 p_{B} \cdot p_{C}\right)\left(2 p_{B} \cdot p_{D}\right)}{s_{\Lambda}^{3}} \mathcal{C}[A] \mathcal{C}[B] \mathcal{C}[C] \mathcal{C}[D], \\
& 1 \in A, \quad B=\Lambda / A, C=\bar{\Lambda} / D, n \in D
\end{aligned}
$$

for the gauge choice $[1, j, \Lambda]$. Or

$$
\begin{aligned}
& I_{\text {org }} \rightarrow \sum_{A \subset \Lambda} \sum_{D \subset \bar{\Lambda}} \frac{\left(2 p_{B} \cdot p_{C}\right)\left(2 p_{A} \cdot p_{C}\right)}{s_{\Lambda}^{3}} \mathcal{C}[A] \mathcal{C}[B] \mathcal{C}[C] \mathcal{C}[D], \\
& 1 \in A, \quad B=\Lambda / A, C=\bar{\Lambda} / D, n \in D
\end{aligned}
$$

for the gauge choice $[i, n, \Lambda]$. Results (4.7) and (4.8) are, in fact, two possible Feynman rules for the triple pole. ${ }^{12}$ It is worth to emphasize that when exchanging the ordering of the sum, the $\sum_{i \in \Lambda \backslash\{1\}} \sum_{j \in \bar{\Lambda} \backslash\{n\}} s_{i j}$ produces $\left(2 p_{B} \cdot p_{C}\right)$. Also, changing of summation ordering is allowed because we have fixed the first gauge choice $1 \in \Lambda, n \in \bar{\Lambda}$ for all splittings of $\langle A B C D\rangle$. The first gauge choice is crucial for the previous Feynman rule, while the second gauge has some natural choice. Since we will use different gauge choices, we will use $[1, n ; 1 ; \Lambda]$ for the gauge choice leading to the rule $(4.7)$ and $[1, n ; n ; \Lambda]$ for the gauge choice leading to the rule (4.8).

\footnotetext{
${ }^{12}$ Again, we need to stick to the same rule for all splittings of $\langle A B C D\rangle$ to get the right result.
} 


\subsection{Comparison with conjectured formula}

The Feynman rules found in the previous subsection is not the one conjectured in [20], which is given by

$$
\begin{aligned}
& \mathcal{R}_{\text {ule }}^{\text {II }}\left[p_{A}, p_{B}, p_{C}, p_{D}\right] \\
& =\frac{\left(2 p_{A} \cdot p_{C}\right)\left(2 p_{A} \cdot p_{D}\right)+\left(2 p_{B} \cdot p_{C}\right)\left(2 p_{B} \cdot p_{D}\right)+\left(2 p_{C} \cdot p_{A}\right)\left(2 p_{C} \cdot p_{B}\right)+\left(2 p_{D} \cdot p_{A}\right)\left(2 p_{D} \cdot p_{B}\right)}{4 s_{A B}^{3}} \\
& \quad-\frac{\left(p_{A}^{2}-p_{B}^{2}\right)^{2}+\left(p_{C}^{2}-p_{D}^{2}\right)^{2}}{4 s_{A B}^{3}}+\frac{2}{9} \frac{\left(p_{A}^{2}+p_{B}^{2}\right)\left(p_{C}^{2}+p_{D}^{2}\right)}{4 s_{A B}^{3}} .
\end{aligned}
$$

Comparing these different Feynman rules, we see that the major difference is that the rule (4.9) is gauge independent, while rules (4.7) and (4.8) depend on both the first gauge choice and the second gauge choice. Because of this, the later two Feynman rules are simpler than the first one. In this subsection, we discuss how to arrive at (4.9) from (4.7) and (4.8).

For simplicity, let us assume that $\Lambda=\{1,2, \ldots, m\}, \bar{\Lambda}=\{m+1, m+2, \ldots, n\}$. The final result of a given $\mathrm{CHY}$ integrand in our case will be the sum of different splittings $\langle A B C D\rangle$

$$
\mathcal{A}=\sum_{1 \in A \subset \Lambda} \sum_{n \in D \subset \bar{\Lambda}} \frac{X}{s_{\Lambda}^{3}} \mathcal{C}[A] \mathcal{C}[B] \mathcal{C}[C] \mathcal{C}[D],
$$

with $B=\Lambda / A$ and $C=\bar{\Lambda} / D$, where to fix the ambiguity, we have set the subset containing node 1 as $A$, and the subset containing node $n$ as $D$. The factor 4 in the denominator (4.9) implies that we should average over four different gauge choices, just like (3.1) is reproduced by average two different gauge choices in previous sections.

Now we consider the following four different gauge choices. For the first gauge choice $[1, n ; 1 ; \Lambda]$, we get

$$
\sum_{1 \in A \subset \Lambda} \sum_{n \in D \subset \bar{\Lambda}} \frac{\left(2 p_{B} \cdot p_{C}\right)\left(2 p_{B} \cdot p_{D}\right)}{s_{\Lambda}^{3}} \mathcal{C}[A] \mathcal{C}[B] \mathcal{C}[C] \mathcal{C}[D],
$$

where the rule (4.7) has been used. For the second gauge choice $[1, n ; n ; \Lambda]$, we get

$$
\sum_{1 \in A \subset \Lambda} \sum_{n \in D \subset \bar{\Lambda}} \frac{\left(2 p_{B} \cdot p_{C}\right)\left(2 p_{A} \cdot p_{C}\right)}{s_{\Lambda}^{3}} \mathcal{C}[A] \mathcal{C}[B] \mathcal{C}[C] \mathcal{C}[D],
$$

where the rule (4.8) has been used. For the third gauge choice $[m ; n ; m ; \Lambda]$, the thing is a little bit complicated: since we have fixed the subset $A$ to be the one containing node 1 , we need to consider two different cases. When $m$ is also in the subset $A$, we get

$$
\sum_{1, m \in A \subset \Lambda} \sum_{n \in D \subset \bar{\Lambda}} \frac{\left(2 p_{B} \cdot p_{C}\right)\left(2 p_{B} \cdot p_{D}\right)}{s_{\Lambda}^{3}} \mathcal{C}[A] \mathcal{C}[B] \mathcal{C}[C] \mathcal{C}[D] .
$$

When $m$ is not in the subset $A$, we get

$$
\sum_{1 \in A \subset \Lambda ; m \notin A} \sum_{n \in D \subset \bar{\Lambda}} \frac{\left(2 p_{A} \cdot p_{C}\right)\left(2 p_{A} \cdot p_{D}\right)}{s_{\Lambda}^{3}} \mathcal{C}[A] \mathcal{C}[B] \mathcal{C}[C] \mathcal{C}[D] .
$$


Adding these two parts (4.13) and (4.14) together, we get the expression for the third gauge choice

$$
\begin{aligned}
& \sum_{1 \in A \subset \Lambda} \sum_{n \in D \subset \bar{\Lambda}} \frac{\left(2 p_{A} \cdot p_{C}\right)\left(2 p_{A} \cdot p_{D}\right)}{s_{\Lambda}^{3}} \mathcal{C}[A] \mathcal{C}[B] \mathcal{C}[C] \mathcal{C}[D] \\
& +\sum_{1, m \in A \subset \Lambda} \sum_{n \in D \subset \bar{\Lambda}} \frac{\left(2 p_{B} \cdot p_{C}\right)\left(2 p_{B} \cdot p_{D}\right)-\left(2 p_{A} \cdot p_{C}\right)\left(2 p_{A} \cdot p_{D}\right)}{s_{\Lambda}^{3}} \mathcal{C}[A] \mathcal{C}[B] \mathcal{C}[C] \mathcal{C}[D]
\end{aligned}
$$

For the fourth gauge choice $[1, m+1 ; m+1 ; \Lambda]$, we need to consider two different cases too. When $(m+1) \in D$, we get

$$
\sum_{1 \in A \subset \Lambda} \sum_{n,(m+1) \in D \subset \bar{\Lambda}} \frac{\left(2 p_{B} \cdot p_{C}\right)\left(2 p_{A} \cdot p_{C}\right)}{s_{\Lambda}^{3}} \mathcal{C}[A] \mathcal{C}[B] \mathcal{C}[C] \mathcal{C}[D]
$$

When $(m+1) \notin D$, we get

$$
\sum_{1 \in A \subset \Lambda} \sum_{n \in D \subset \bar{\Lambda} ;(m+1) \notin D} \frac{\left(2 p_{D} \cdot p_{A}\right)\left(2 p_{D} \cdot p_{B}\right)}{s_{\Lambda}^{3}} \mathcal{C}[A] \mathcal{C}[B] \mathcal{C}[C] \mathcal{C}[D]
$$

Summing over (4.16) and (4.17), the expression of the fourth gauge choice is

$$
\begin{aligned}
& \sum_{1 \in A \subset \Lambda} \sum_{n \in D \subset \bar{\Lambda}} \frac{\left(2 p_{D} \cdot p_{A}\right)\left(2 p_{D} \cdot p_{B}\right)}{s_{\Lambda}^{3}} \mathcal{C}[A] \mathcal{C}[B] \mathcal{C}[C] \mathcal{C}[D] \\
& +\sum_{1 \in A \subset \Lambda} \sum_{n,(m+1) \in D \subset \bar{\Lambda}} \frac{\left(2 p_{B} \cdot p_{C}\right)\left(2 p_{A} \cdot p_{C}\right)-\left(2 p_{D} \cdot p_{A}\right)\left(2 p_{D} \cdot p_{B}\right)}{s_{\Lambda}^{3}} \mathcal{C}[A] \mathcal{C}[B] \mathcal{C}[C] \mathcal{C}[D]
\end{aligned}
$$

Now we average four different gauge choices (4.11), (4.12), (4.15) and (4.18) to reach $X$ in $(4.10)$ as

$$
\begin{aligned}
\mathcal{A}= & \sum_{\langle A B C D\rangle} \bar{X} \mathcal{C}[A] \mathcal{C}[B] \mathcal{C}[C] \mathcal{C}[D] \\
& +\sum_{1 \in A \subset \Lambda} \sum_{n,(m+1) \in D \subset \bar{\Lambda}} \frac{\left(2 p_{B} \cdot p_{C}\right)\left(2 p_{A} \cdot p_{C}\right)-\left(2 p_{D} \cdot p_{A}\right)\left(2 p_{D} \cdot p_{B}\right)}{4} \mathcal{C}[A] \mathcal{C}[B] \mathcal{C}[C] \mathcal{C}[D] \\
& +\sum_{1, m \in A \subset \Lambda} \sum_{n \in D \subset \bar{\Lambda}} \frac{\left(2 p_{B} \cdot p_{C}\right)\left(2 p_{B} \cdot p_{D}\right)-\left(2 p_{A} \cdot p_{C}\right)\left(2 p_{A} \cdot p_{D}\right)}{4} \mathcal{C}[A] \mathcal{C}[B] \mathcal{C}[C] \mathcal{C}[D]
\end{aligned}
$$

where

$$
\bar{X}=\frac{\left(2 p_{A} \cdot p_{C}\right)\left(2 p_{A} \cdot p_{D}\right)+\left(2 p_{B} \cdot p_{C}\right)\left(2 p_{B} \cdot p_{D}\right)+\left(2 p_{C} \cdot p_{A}\right)\left(2 p_{C} \cdot p_{B}\right)+\left(2 p_{D} \cdot p_{A}\right)\left(2 p_{D} \cdot p_{B}\right)}{4} .
$$


Thus to reproduce the rule (4.9), we need to show that

$$
\begin{aligned}
& \sum_{1 \in A \subset \Lambda} \sum_{n \in D \subset \bar{\Lambda}}-\frac{\left(p_{A}^{2}-p_{B}^{2}\right)^{2}+\left(p_{C}^{2}-p_{D}^{2}\right)^{2}+\frac{2}{9}\left(p_{A}^{2}+p_{B}^{2}\right)\left(p_{C}^{2}+p_{D}^{2}\right)}{4 s_{\Lambda}^{3}} \mathcal{C}[A] \mathcal{C}[B] \mathcal{C}[C] \mathcal{C}[D] \\
& =\sum_{1 \in A \subset \Lambda} \sum_{n,(m+1) \in D \subset \bar{\Lambda}} \frac{\left(2 p_{B} \cdot p_{C}\right)\left(2 p_{A} \cdot p_{C}\right)-\left(2 p_{D} \cdot p_{A}\right)\left(2 p_{D} \cdot p_{B}\right)}{4 s_{\Lambda}^{3}} \mathcal{C}[A] \mathcal{C}[B] \mathcal{C}[C] \mathcal{C}[D] \\
& \quad+\sum_{1, m \in A \subset \Lambda} \sum_{n \in D \subset \bar{\Lambda}} \frac{\left(2 p_{B} \cdot p_{C}\right)\left(2 p_{B} \cdot p_{D}\right)-\left(2 p_{A} \cdot p_{C}\right)\left(2 p_{A} \cdot p_{D}\right)}{4 s_{\Lambda}^{3}} \mathcal{C}[A] \mathcal{C}[B] \mathcal{C}[C] \mathcal{C}[D]
\end{aligned}
$$

To make the comparison explicitly, we rewrite $\left(2 p_{B} \cdot p_{C}\right)\left(2 p_{B} \cdot p_{D}\right)-\left(2 p_{A} \cdot p_{C}\right)\left(2 p_{A} \cdot p_{D}\right)$ as follows

$$
\begin{aligned}
( & \left.2 p_{B} \cdot p_{C}\right)\left(2 p_{B} \cdot p_{D}\right)-\left(2 p_{A} \cdot p_{C}\right)\left(2 p_{A} \cdot p_{D}\right) \\
= & \left(2 p_{B} \cdot p_{C}\right)\left(2 p_{B} \cdot p_{D}\right)-\left(2 p_{\Lambda} \cdot p_{C}\right)\left(2 p_{\Lambda} \cdot p_{D}\right)+\left(2 p_{B} \cdot p_{C}\right)\left(2 p_{\Lambda} \cdot p_{D}\right)+\left(2 p_{\Lambda} \cdot p_{C}\right)\left(2 p_{B} \cdot p_{D}\right) \\
& -\left(2 p_{B} \cdot p_{C}\right)\left(2 p_{B} \cdot p_{D}\right) \\
= & -\left(2 p_{\bar{\Lambda}} \cdot p_{C}\right)\left(2 p_{\bar{\Lambda}} \cdot p_{D}\right)-\left(2 p_{B} \cdot p_{C}\right)\left(2 p_{\bar{\Lambda}} \cdot p_{D}\right)-\left(2 p_{\bar{\Lambda}} \cdot p_{C}\right)\left(2 p_{B} \cdot p_{D}\right) \\
= & -\left(2 s_{C}+2 p_{C} \cdot p_{D}\right)\left(2 s_{D}+2 p_{C} \cdot p_{D}\right)-\left(2 p_{B} \cdot p_{C}\right)\left(2 s_{D}+2 p_{C} \cdot p_{D}\right)-\left(2 p_{B} \cdot p_{D}\right)\left(2 s_{C}+2 p_{C} \cdot p_{D}\right) \\
= & -\left(2 p_{C} \cdot p_{D}\right)\left(s_{C}+s_{D}+2 p_{C} \cdot p_{D}+2 p_{B} \cdot p_{C}+2 p_{B} \cdot p_{D}\right)-4 s_{C} s_{D}-s_{C}\left(2 p_{C} \cdot p_{D}+4 p_{B} \cdot p_{D}\right) \\
& -s_{D}\left(2 p_{C} \cdot p_{D}+4 p_{B} \cdot p_{C}\right) \\
= & -\left(2 p_{C} \cdot p_{D}\right)\left(s_{A}-s_{B}\right)-4 s_{C} s_{D}-s_{C}\left(2 p_{C} \cdot p_{D}+4 p_{B} \cdot p_{D}\right)-s_{D}\left(2 p_{C} \cdot p_{D}+4 p_{B} \cdot p_{C}\right) \\
= & -s_{\Lambda}\left(s_{A}-s_{B}\right)+\mathcal{K}_{1},
\end{aligned}
$$

where

$\mathcal{K}_{1}=-4 s_{C} s_{D}-s_{C}\left(2 p_{C} \cdot p_{D}+4 p_{B} \cdot p_{D}+s_{B}-s_{A}\right)-s_{D}\left(2 p_{C} \cdot p_{D}+4 p_{B} \cdot p_{C}+s_{B}-s_{A}\right)$,

and similarly

$$
\left(2 p_{A} \cdot p_{C}\right)\left(2 p_{B} \cdot p_{C}\right)-\left(2 p_{A} \cdot p_{D}\right)\left(2 p_{B} \cdot p_{D}\right)=-s_{\bar{\Lambda}}\left(s_{D}-s_{C}\right)+\mathcal{K}_{2}
$$

where

$\mathcal{K}_{2}=-4 s_{A} s_{B}-s_{A}\left(2 p_{A} \cdot p_{B}+4 p_{C} \cdot p_{B}+s_{C}-s_{D}\right)-s_{B}\left(2 p_{A} \cdot p_{B}+4 p_{C} \cdot p_{A}+s_{C}-s_{D}\right)$.

We will prove that

$$
\begin{aligned}
\sum_{1, m \in A \subset \Lambda} s_{\Lambda}\left(s_{A}-s_{B}\right) \mathcal{C}[A] \mathcal{C}[B] & =\sum_{1 \in A \subset \Lambda}\left(s_{A}-s_{B}\right)^{2} \mathcal{C}[A] \mathcal{C}[B], \\
\sum_{m+1, n \in D \subset \Lambda} s_{\bar{\Lambda}}\left(s_{D}-s_{C}\right) \mathcal{C}[C] \mathcal{C}[D] & =\sum_{n \in D \subset \Lambda}\left(s_{C}-s_{D}\right)^{2} \mathcal{C}[C] \mathcal{C}[D],
\end{aligned}
$$



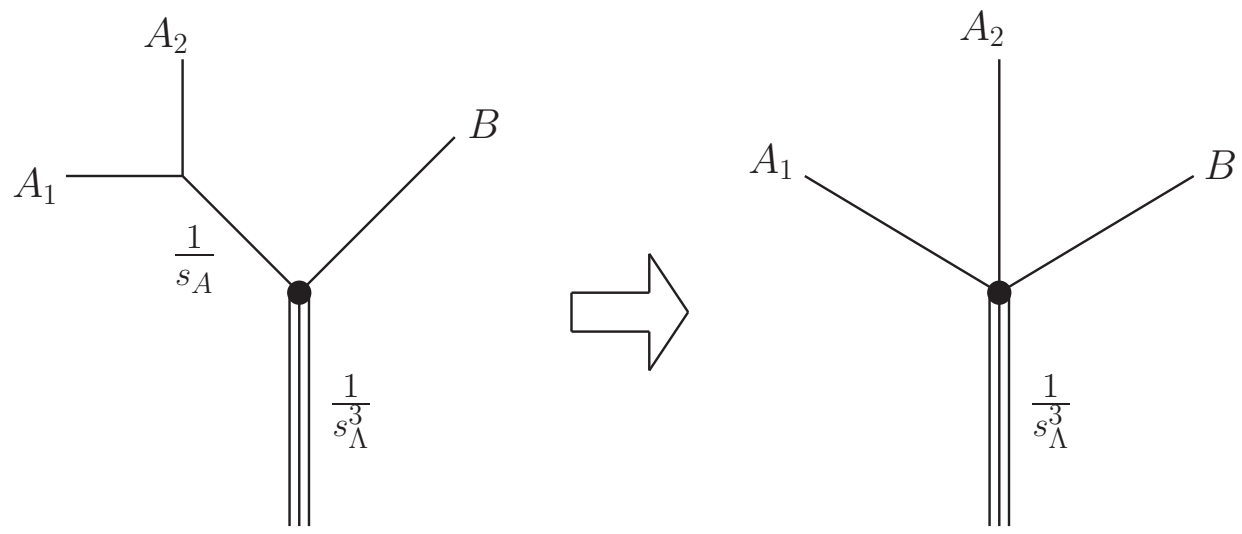

Figure 3. Creation of the quartic vertex from a cubic vertex.

and

$$
\begin{aligned}
& \sum_{1, m \in A \subset \Lambda} \sum_{n \in D \subset \bar{\Lambda}} \mathcal{K}_{1} \mathcal{C}[A] \mathcal{C}[B] \mathcal{C}[C] \mathcal{C}[D] \\
& =\sum_{1 \in A \subset \Lambda} \sum_{n,(m+1) \in D \subset \bar{\Lambda}} \mathcal{K}_{2} \mathcal{C}[A] \mathcal{C}[B] \mathcal{C}[C] \mathcal{C}[D] \\
& =\sum_{1 \in A \subset \Lambda} \sum_{n \in D \subset \bar{\Lambda}} \frac{1}{9}\left(s_{A}+s_{B}\right)\left(s_{C}+s_{D}\right) \mathcal{C}[A] \mathcal{C}[B] \mathcal{C}[C] \mathcal{C}[D]
\end{aligned}
$$

Putting (4.26), (4.27) and (4.28) back to (4.21), we see the identity is proved.

Let us start with the relation (4.26). At the left handed side, since we have assumed that $A$ contains at least two points 1 and $m$, using (2.5) we know that $s_{A} \mathcal{C}[A] \mathcal{C}[B]$ will remove the single pole $\frac{1}{s_{A}}$ inside $\mathcal{C}[A]$ and split to $\sum_{\left\langle A_{1} A_{2}\right\rangle} \mathcal{C}\left[A_{1}\right] \mathcal{C}\left[A_{2}\right]$ with $A=A_{1} \bigcup A_{2}$. This procedure removes the propagator $1 / s_{A}$ from the cubic vertex, and creates a new quartic vertex by pushing $A_{1}$ and $A_{2}$ to the original cubic vertex, as shown in figure 3. Similarly, if $B$ also contains at least two points, $s_{B} \mathcal{C}[B]=\sum_{\left\langle B_{1} B_{2}\right\rangle} \mathcal{C}\left[B_{1}\right] \mathcal{C}\left[B_{2}\right]$ with $B=B_{1} \bigcup B_{2}$. In other words, we will have

$$
\left(s_{A}-s_{B}\right) \mathcal{C}[A] \mathcal{C}[B]=\sum_{\left\langle A_{1} A_{2}\right\rangle} \mathcal{C}\left[A_{1}\right] \mathcal{C}\left[A_{2}\right] \mathcal{C}[B]-\sum_{\left\langle B_{1} B_{2}\right\rangle} \mathcal{C}[A] \mathcal{C}\left[B_{1}\right] \mathcal{C}\left[B_{2}\right]
$$

where to fix the ambiguity we will assume that $1 \in A_{1}$. The summation in the first term is over all correct divisions of $A_{1}$ and $A_{2}$, and the summation in the second term is over all correct divisions of $B_{1}$ and $B_{2}$. We emphasize that a correct division must ensure that $A_{1}, A_{2}$, $B_{1}$ and $B_{2}$ are simple sets since they corresponding to Feynman lines in Feynman diagrams.

If $B$ contains only one point, we have

$$
\left(s_{A}-s_{B}\right) \mathcal{C}[A] \mathcal{C}[B]=\sum_{\left\langle A_{1} A_{2}\right\rangle} \mathcal{C}\left[A_{1}\right] \mathcal{C}\left[A_{2}\right] \mathcal{C}[B]
$$

The discussion above implies that $\sum\left(s_{A}-s_{B}\right) \mathcal{C}[A] \mathcal{C}[B]$ with $1, m \in A$ will be the summation of several $\mathcal{C}\left[\Lambda_{1}\right] \mathcal{C}\left[\Lambda_{2}\right] \mathcal{C}\left[\Lambda_{3}\right]$ 's, where $\Lambda_{1}, \Lambda_{2}$ and $\Lambda_{3}$ are simple sets satisfy $\Lambda_{1} \bigcup \Lambda_{2} \bigcup \Lambda_{3}=\Lambda$. 
We fix the configuration as $1 \in \Lambda_{1}$. Thus we can consider the coefficient of $\mathcal{C}\left[\Lambda_{1}\right] \mathcal{C}\left[\Lambda_{2}\right] \mathcal{C}\left[\Lambda_{3}\right]$ under a special division $\left\langle\Lambda_{1} \Lambda_{2} \Lambda_{3}\right\rangle$. There are several cases:

(1) If $m \in \Lambda_{1}$, there are three correct configurations which are $\left\{A_{1}=\Lambda_{1}, A_{2}=\Lambda_{2}, B=\Lambda_{3}\right\}$, $\left\{A_{1}=\Lambda_{1}, A_{2}=\Lambda_{3}, B=\Lambda_{2}\right\}$ and $\left\{A=\Lambda_{1}, B_{1}=\Lambda_{2}, B_{2}=\Lambda_{3}\right\}$, thus, after summing over all correct divisions, the coefficient of $\mathcal{C}\left[\Lambda_{1}\right] \mathcal{C}\left[\Lambda_{2}\right] \mathcal{C}\left[\Lambda_{3}\right]$ is $1+1-1=1$. Notice that for the third configuration above, $\left\{A=\Lambda_{1}, B_{1}=\Lambda_{3}, B_{2}=\Lambda_{2}\right\}$ gives the same situation $B=\Lambda_{2} \bigcup \Lambda_{3}$ as $\left\{A=\Lambda_{1}, B_{1}=\Lambda_{2}, B_{2}=\Lambda_{3}\right\}$, so we chose only one of them. An alternative way is to add them together and divide by the symmetry factor 2 .

(2) If $m \in \Lambda_{2}\left(m \in \Lambda_{3}\right.$ gives the same situation), there is only one correct configuration $\left\{A_{1}=\Lambda_{1}, A_{2}=\Lambda_{2}, B=\Lambda_{3}\right\}$, thus the coefficient is 1 .

Thus, the coefficient of any $\mathcal{C}\left[\Lambda_{1}\right] \mathcal{C}\left[\Lambda_{2}\right] \mathcal{C}\left[\Lambda_{3}\right]$ is 1 , we get

$$
\sum_{1, m \in A \subset \Lambda} s_{\Lambda}\left(s_{A}-s_{B}\right) \mathcal{C}[A] \mathcal{C}[B]=s_{\Lambda} \sum_{\left\langle\Lambda_{1} \Lambda_{2} \Lambda_{3}\right\rangle} \mathcal{C}\left[\Lambda_{1}\right] \mathcal{C}\left[\Lambda_{2}\right] \mathcal{C}\left[\Lambda_{3}\right]
$$

One may observe that (4.30) can be ignored when counting the coefficient of $\mathcal{C}\left[\Lambda_{1}\right] \mathcal{C}\left[\Lambda_{2}\right] \mathcal{C}\left[\Lambda_{3}\right]$. The reason is, if we consider the configuration $\left\{A=\Lambda_{1}, B_{1}=\Lambda_{2}, B_{2}=\Lambda_{3}\right\}$, we have assumed that $B$ contains at least two points. If we consider other configurations, the structure of $B$ is not important. The set $\Lambda$ can always be divided into three simple subsets if it contains at least three points. We can take the perspective that $A$ and $B$ are constructed by three subsets, then the irrelevant cases (such as $B$ can not be divided into two simple subsets) will be neglected automatically.

Having considered the left handed side of relation (4.26), we move to the right handed side, i.e., the $\left(s_{A}-s_{B}\right)^{2} \mathcal{C}[A] \mathcal{C}[B]$ part. By similar argument, we can write it as

$$
\left(s_{A}-s_{B}\right)^{2} \mathcal{C}[A] \mathcal{C}[B]=\left(s_{A}-s_{B}\right)\left(\sum_{\left\langle A_{1} A_{2}\right\rangle} \mathcal{C}\left[A_{1}\right] \mathcal{C}\left[A_{2}\right] \mathcal{C}[B]-\sum_{\left\langle B_{1} B_{2}\right\rangle} \mathcal{C}[A] \mathcal{C}\left[B_{1}\right] \mathcal{C}\left[B_{2}\right]\right)
$$

If one of $A$ and $B$ is a single point, one term in the bracket vanishes. Thus $\sum\left(s_{A}-s_{B}\right)^{2} \mathcal{C}[A] \mathcal{C}[B]$ also provides several $\mathcal{C}\left[\Lambda_{1}\right] \mathcal{C}\left[\Lambda_{2}\right] \mathcal{C}\left[\Lambda_{3}\right]^{\prime}$ s. Let us count the coefficient of $\mathcal{C}\left[\Lambda_{1}\right] \mathcal{C}\left[\Lambda_{2}\right] \mathcal{C}\left[\Lambda_{3}\right]$ with $1 \in \Lambda_{1}$, under the summation $\sum\left(s_{A}-s_{B}\right)^{2} \mathcal{C}[A] \mathcal{C}[B]$ which is over all divisions $\langle A B\rangle$, without the constraint $m \in A$. There are three correct configurations, $\left\{A_{1}=\Lambda_{1}, A_{2}=\Lambda_{2}, B=\Lambda_{3}\right\},\left\{A_{1}=\Lambda_{1}, A_{2}=\Lambda_{3}, B=\Lambda_{2}\right\}$ and $\left\{A=\Lambda_{1}, B_{1}=\Lambda_{2}, B_{2}=\Lambda_{3}\right\}$, then the coefficient is

$$
\left(s_{\Lambda_{1} \Lambda_{2}}-s_{\Lambda_{3}}\right)+\left(s_{\Lambda_{1} \Lambda_{3}}-s_{\Lambda_{2}}\right)-\left(s_{\Lambda_{1}}-s_{\Lambda_{2} \Lambda_{3}}\right)=s_{\Lambda_{1} \Lambda_{2} \Lambda_{3}}=s_{\Lambda} .
$$

Notice that if some of $\Lambda_{i}$ 's are single points, i.e., $s_{\Lambda_{i}}=0$, the above relation still holds, as can be verified directly. Thus, after summing over contributions from different divisions $\langle A B\rangle$, We get

$$
\sum_{1, m \in A \subset \Lambda} s_{\Lambda}\left(s_{A}-s_{B}\right) \mathcal{C}[A] \mathcal{C}[B]=\sum_{1 \in A \subset \Lambda}\left(s_{A}-s_{B}\right)^{2} \mathcal{C}[A] \mathcal{C}[B]=s_{\Lambda} \sum_{\left\langle\Lambda_{1} \Lambda_{2} \Lambda_{3}\right\rangle} \mathcal{C}\left[\Lambda_{1}\right] \mathcal{C}\left[\Lambda_{2}\right] \mathcal{C}\left[\Lambda_{3}\right],
$$

which has proven the relation (4.26). 
Exactly similar argument shows

$\sum_{m+1, n \in D \subset \Lambda} s_{\bar{\Lambda}}\left(s_{D}-s_{C}\right) \mathcal{C}[C] \mathcal{C}[D]=\sum_{n \in D \subset \Lambda}\left(s_{C}-s_{D}\right)^{2} \mathcal{C}[C] \mathcal{C}[D]=s_{\bar{\Lambda}} \sum_{\left\langle\bar{\Lambda}_{1} \bar{\Lambda}_{2} \bar{\Lambda}_{3}\right\rangle} \mathcal{C}\left[\bar{\Lambda}_{1}\right] \mathcal{C}\left[\bar{\Lambda}_{2}\right] \mathcal{C}\left[\bar{\Lambda}_{3}\right]$

where $\bar{\Lambda}_{1}, \bar{\Lambda}_{2}$ and $\bar{\Lambda}_{3}$ are three simple sets with $\bar{\Lambda}_{1} \cup \bar{\Lambda}_{2} \bigcup \bar{\Lambda}_{3}=\bar{\Lambda}$, which has proved the relation (4.27).

Then we turn to the most difficult relation (4.28). We divide $\sum \mathcal{K}_{1} \mathcal{C}[A] \mathcal{C}[B] \mathcal{C}[C] \mathcal{C}[D]$ into following three parts:

$\begin{aligned} Y_{1} & =\sum_{1, m \in A \subset \Lambda} \sum_{n \in D \subset \bar{\Lambda}}-\left(s_{C}+s_{D}\right)\left(s_{B}-s_{A}\right) \mathcal{C}[A] \mathcal{C}[B] \mathcal{C}[C] \mathcal{C}[D] \\ Y_{2} & =\sum_{1, m \in A \subset \Lambda} \sum_{n \in D \subset \bar{\Lambda}}-\left(s_{C}\left(2 p_{C} \cdot p_{D}+4 p_{B} \cdot p_{D}\right)+s_{D}\left(2 p_{C} \cdot p_{D}+4 p_{B} \cdot p_{C}\right)\right) \mathcal{C}[A] \mathcal{C}[B] \mathcal{C}[C] \mathcal{C}[D]\end{aligned}$

$Y_{3}=\sum_{1, m \in A \subset \Lambda} \sum_{n \in D \subset \bar{\Lambda}}-4 s_{C} s_{D} \mathcal{C}[A] \mathcal{C}[B] \mathcal{C}[C] \mathcal{C}[D]$

and will treat them one by one.

First, using (4.31) we get

$$
\begin{aligned}
Y_{1} & =\sum_{n \in D \subset \bar{\Lambda}}\left(s_{C}+s_{D}\right) \mathcal{C}[C] \mathcal{C}[D] \sum_{\left\langle\Lambda_{1} \Lambda_{2} \Lambda_{3}\right\rangle} \mathcal{C}\left[\Lambda_{1}\right] \mathcal{C}\left[\Lambda_{2}\right] \mathcal{C}\left[\Lambda_{3}\right] \\
& =\sum_{n \in D \subset \bar{\Lambda}}\left(\sum_{\left\langle C_{1} C_{2}\right\rangle} \mathcal{C}\left[C_{1}\right] \mathcal{C}\left[C_{2}\right] \mathcal{C}[D]+\sum_{\left\langle D_{1} D_{2}\right\rangle} \mathcal{C}[C] \mathcal{C}\left[D_{1}\right] \mathcal{C}\left[D_{2}\right]\right) \sum_{\left\langle\Lambda_{1} \Lambda_{2} \Lambda_{3}\right\rangle} \mathcal{C}\left[\Lambda_{1}\right] \mathcal{C}\left[\Lambda_{2}\right] \mathcal{C}\left[\Lambda_{3}\right]
\end{aligned}
$$

We assume that $n \in D_{1}$. We can consider the coefficient of $\mathcal{C}\left[\Lambda_{1}\right] \mathcal{C}\left[\Lambda_{2}\right] \mathcal{C}\left[\Lambda_{3}\right] \mathcal{C}\left[\bar{\Lambda}_{1}\right] \mathcal{C}\left[\bar{\Lambda}_{2}\right] \mathcal{C}\left[\bar{\Lambda}_{2}\right]$ with $n \in \bar{\Lambda}_{1}$. There are three correct configurations which are $\left\{D_{1}=\bar{\Lambda}_{1}, D_{2}=\bar{\Lambda}_{2}, C=\bar{\Lambda}_{3}\right\}$, $\left\{D_{1}=\bar{\Lambda}_{1}, D_{2}=\bar{\Lambda}_{3}, D=\bar{\Lambda}_{2}\right\}$ and $\left\{D=\bar{\Lambda}_{1}, C_{1}=\bar{\Lambda}_{2}, C_{2}=\bar{\Lambda}_{3}\right\}$, thus we have

$$
Y_{1}=3 \sum_{\left\langle\Lambda_{1} \Lambda_{2} \Lambda_{3}\right\rangle} \sum_{\left\langle\bar{\Lambda}_{1} \bar{\Lambda}_{2} \bar{\Lambda}_{3}\right\rangle}\left(\mathcal{C}\left[\Lambda_{1}\right] \mathcal{C}\left[\Lambda_{2}\right] \mathcal{C}\left[\Lambda_{3}\right]\right)\left(\mathcal{C}\left[\bar{\Lambda}_{1}\right] \mathcal{C}\left[\bar{\Lambda}_{2}\right] \mathcal{C}\left[\bar{\Lambda}_{3}\right]\right)
$$

Secondly, considering $Y_{2}$ gives

$$
\begin{aligned}
Y_{2}= & \sum_{1, m \in A \subset \Lambda} \sum_{n \in D \subset \bar{\Lambda}}-\left(\left(2 p_{C} \cdot p_{D}+4 p_{B} \cdot p_{D}\right) \sum_{\left\langle C_{1} C_{2}\right\rangle} \mathcal{C}\left[C_{1}\right] \mathcal{C}\left[C_{2}\right] \mathcal{C}[D]\right. \\
& \left.+\left(2 p_{C} \cdot p_{D}+4 p_{B} \cdot p_{C}\right) \sum_{\left\langle D_{1} D_{2}\right\rangle} \mathcal{C}[C] \mathcal{C}\left[D_{1}\right] \mathcal{C}\left[D_{2}\right]\right) \mathcal{C}[A] \mathcal{C}[B]
\end{aligned}
$$

Now we fix $A, B$, and count the coefficient of $\mathcal{C}\left[\bar{\Lambda}_{1}\right] \mathcal{C}\left[\bar{\Lambda}_{2}\right] \mathcal{C}\left[\bar{\Lambda}_{3}\right]$ with $n \in \bar{\Lambda}_{1}$ under the summation over divisions $\langle C D\rangle$. The correct configurations have been given when considering 
$Y_{1}$, thus the coefficient is

$$
\begin{aligned}
& -\left(2 p_{\bar{\Lambda}_{1} \bar{\Lambda}_{2}} \cdot p_{\bar{\Lambda}_{3}}+4 p_{\bar{\Lambda}_{3}} \cdot p_{B}+2 p_{\bar{\Lambda}_{1} \bar{\Lambda}_{3}} \cdot p_{\bar{\Lambda}_{2}}+4 p_{\bar{\Lambda}_{2}} \cdot p_{B}+2 p_{\bar{\Lambda}_{2} \bar{\Lambda}_{3}} \cdot p_{\bar{\Lambda}_{1}}+4 p_{\bar{\Lambda}_{1}} \cdot p_{B}\right) \\
& \quad=-2\left(s_{A}-s_{B}\right)+2 s_{\bar{\Lambda}_{1}}+2 s_{\bar{\Lambda}_{2}}+2 s_{\bar{\Lambda}_{3}} .
\end{aligned}
$$

Substituting it into (4.41) we get

$$
\begin{aligned}
Y_{2}= & \sum_{1, m \in A \subset \Lambda} \sum_{\left\langle\bar{\Lambda}_{1} \bar{\Lambda}_{2} \bar{\Lambda}_{3}\right\rangle}\left(-2\left(s_{A}-s_{B}\right)+2 s_{\bar{\Lambda}_{1}}+2 s_{\bar{\Lambda}_{2}}+2 s_{\bar{\Lambda}_{3}}\right)(\mathcal{C}[A] \mathcal{C}[B])\left(\mathcal{C}\left[\bar{\Lambda}_{1}\right] \mathcal{C}\left[\bar{\Lambda}_{2}\right] \mathcal{C}\left[\bar{\Lambda}_{3}\right]\right) \\
= & -2 \sum_{\left\langle\Lambda_{1} \Lambda_{2} \Lambda_{3}\right\rangle} \sum_{\left\langle\bar{\Lambda}_{1} \bar{\Lambda}_{2} \bar{\Lambda}_{3}\right\rangle}\left(\mathcal{C}\left[\Lambda_{1}\right] \mathcal{C}\left[\Lambda_{2}\right] \mathcal{C}\left[\Lambda_{3}\right]\right)\left(\mathcal{C}\left[\bar{\Lambda}_{1}\right] \mathcal{C}\left[\bar{\Lambda}_{2}\right] \mathcal{C}\left[\bar{\Lambda}_{3}\right]\right) \\
& +2 \sum_{1, m \in A \subset \Lambda} \mathcal{C}[A] \mathcal{C}[B] \sum_{\left\langle\bar{\Lambda}_{1} \bar{\Lambda}_{2} \bar{\Lambda}_{3}\right\rangle}\left(\sum_{\left\langle\bar{\Lambda}_{11} \bar{\Lambda}_{12}\right\rangle} \mathcal{C}\left[\bar{\Lambda}_{11}\right] \mathcal{C}\left[\bar{\Lambda}_{12}\right] \mathcal{C}\left[\bar{\Lambda}_{2}\right] \mathcal{C}\left[\bar{\Lambda}_{3}\right]\right. \\
& \left.+\sum_{\left\langle\bar{\Lambda}_{21} \bar{\Lambda}_{22}\right\rangle} \mathcal{C}\left[\bar{\Lambda}_{1}\right] \mathcal{C}\left[\bar{\Lambda}_{21}\right] \mathcal{C}\left[\bar{\Lambda}_{22}\right] \mathcal{C}\left[\bar{\Lambda}_{3}\right]+\sum_{\left\langle\bar{\Lambda}_{31} \bar{\Lambda}_{32}\right\rangle} \mathcal{C}\left[\bar{\Lambda}_{1}\right] \mathcal{C}\left[\bar{\Lambda}_{2}\right] \mathcal{C}\left[\bar{\Lambda}_{31}\right] \mathcal{C}\left[\bar{\Lambda}_{32}\right]\right)
\end{aligned}
$$

where (4.31) has been used again. In the last line, $\bar{\Lambda}_{i 1}$ and $\bar{\Lambda}_{i 2}$ are two simple subsets of $\bar{\Lambda}_{i}$. For the term $\mathcal{C}\left[\lambda_{1}\right] \mathcal{C}\left[\lambda_{2}\right] \mathcal{C}\left[\lambda_{3}\right] \mathcal{C}\left[\lambda_{4}\right]$ with $n \in \lambda_{1}$ and $\lambda_{1} \cup \lambda_{2} \cup \lambda_{3} \cup \lambda_{4}=\bar{\Lambda}$, its coefficient under the summation over divisions $\left\langle\bar{\Lambda}_{1} \bar{\Lambda}_{2} \bar{\Lambda}_{3}\right\rangle$ can be counted by following six configurations: $\left\{\bar{\Lambda}_{11}=\lambda_{1}, \bar{\Lambda}_{12}=\lambda_{2}, \bar{\Lambda}_{2}=\lambda_{3}, \bar{\Lambda}_{3}=\lambda_{4}\right\},\left\{\bar{\Lambda}_{11}=\lambda_{1}, \bar{\Lambda}_{12}=\lambda_{3}, \bar{\Lambda}_{2}=\lambda_{2}, \bar{\Lambda}_{3}=\lambda_{4}\right\}$, $\left\{\bar{\Lambda}_{11}=\lambda_{1}, \bar{\Lambda}_{12}=\lambda_{4}, \bar{\Lambda}_{2}=\lambda_{2}, \bar{\Lambda}_{3}=\lambda_{3}\right\},\left\{\bar{\Lambda}_{1}=\lambda_{1}, \bar{\Lambda}_{21}=\lambda_{2}, \bar{\Lambda}_{22}=\lambda_{3}, \bar{\Lambda}_{3}=\lambda_{4}\right\}$, $\left\{\bar{\Lambda}_{1}=\lambda_{1}, \bar{\Lambda}_{21}=\lambda_{2}, \bar{\Lambda}_{22}=\lambda_{4}, \bar{\Lambda}_{3}=\lambda_{3}\right\},\left\{\bar{\Lambda}_{1}=\lambda_{1}, \bar{\Lambda}_{21}=\lambda_{3}, \bar{\Lambda}_{22}=\lambda_{4}, \bar{\Lambda}_{3}=\lambda_{2}\right\}$. Thus, we find

$$
\begin{aligned}
Y_{2}= & -2 \sum_{\left\langle\Lambda_{1} \Lambda_{2} \Lambda_{3}\right\rangle} \sum_{\left\langle\bar{\Lambda}_{1} \bar{\Lambda}_{2} \bar{\Lambda}_{3}\right\rangle}\left(\mathcal{C}\left[\Lambda_{1}\right] \mathcal{C}\left[\Lambda_{2}\right] \mathcal{C}\left[\Lambda_{3}\right]\right)\left(\mathcal{C}\left[\bar{\Lambda}_{1}\right] \mathcal{C}\left[\bar{\Lambda}_{2}\right] \mathcal{C}\left[\bar{\Lambda}_{3}\right]\right) \\
& +12 \sum_{1, m \in A \subset \Lambda} \mathcal{C}[A] \mathcal{C}[B] \sum_{\left\langle\lambda_{1} \lambda_{2} \lambda_{3} \lambda_{4}\right\rangle} \mathcal{C}\left[\lambda_{1}\right] \mathcal{C}\left[\lambda_{2}\right] \mathcal{C}\left[\lambda_{3}\right] \mathcal{C}\left[\lambda_{4}\right]
\end{aligned}
$$

Similar to the appearing of quartic vertexes, the term $\mathcal{C}\left[\lambda_{1}\right] \mathcal{C}\left[\lambda_{2}\right] \mathcal{C}\left[\lambda_{3}\right] \mathcal{C}\left[\lambda_{4}\right]$ can be explained as a new quintic vertex.

Finally, we consider $Y_{3}$ to obtain

$$
Y_{3}=-4 \sum_{1, m \in A \subset \Lambda} \mathcal{C}[A] \mathcal{C}[B] \sum_{n \in D \subset \bar{\Lambda}}\left(\sum_{\left\langle C_{1} C_{2}\right\rangle} \mathcal{C}\left[C_{1}\right] \mathcal{C}\left[C_{2}\right]\right)\left(\sum_{\left\langle D_{1} D_{2}\right\rangle} \mathcal{C}\left[D_{1}\right] \mathcal{C}\left[D_{2}\right]\right)
$$

where $C_{1}$ and $C_{2}$ are two simple subsets of $C, D_{1}$ and $D_{2}$ are two simple subsets of $D$ with $n \in D_{1}$. Considering the coefficient of $\mathcal{C}\left[\lambda_{1}\right] \mathcal{C}\left[\lambda_{2}\right] \mathcal{C}\left[\lambda_{3}\right] \mathcal{C}\left[\lambda_{4}\right]$ with $n \in \lambda_{1}$ under the summation over divisions $\langle C D\rangle$, one can find three correct configurations: $\left\{D_{1}=\lambda_{1}, D_{2}=\lambda_{2}, C_{1}=\lambda_{3}, C_{2}=\lambda_{4}\right\},\left\{D_{1}=\lambda_{1}, D_{2}=\lambda_{3}, C_{1}=\lambda_{2}, C_{2}=\lambda_{4}\right\}$ and $\left\{D_{1}=\lambda_{1}, D_{2}=\lambda_{4}, C_{1}=\lambda_{2}, C_{2}=\lambda_{3}\right\}$, thus we arrive at

$$
Y_{3}=-12 \sum_{1, m \in A \subset \Lambda} \mathcal{C}[A] \mathcal{C}[B] \sum_{\left\langle\lambda_{1} \lambda_{2} \lambda_{3} \lambda_{4}\right\rangle} \mathcal{C}\left[\lambda_{1}\right] \mathcal{C}\left[\lambda_{2}\right] \mathcal{C}\left[\lambda_{3}\right] \mathcal{C}\left[\lambda_{4}\right]
$$


Adding $Y_{1}, Y_{2}$ and $Y_{3}$ together, we can see that the quintic vertexes cancels each other, and the result is given as

$$
\begin{aligned}
& \sum_{1, m \in A \subset \Lambda} \sum_{n \in D \subset \bar{\Lambda}} \mathcal{K}_{1} \mathcal{C}[A] \mathcal{C}[B] \mathcal{C}[C] \mathcal{C}[D] \\
& =\sum_{\left\langle\Lambda_{1} \Lambda_{2} \Lambda_{3}\right\rangle} \sum_{\left\langle\bar{\Lambda}_{1} \bar{\Lambda}_{2} \bar{\Lambda}_{3}\right\rangle}\left(\mathcal{C}\left[\Lambda_{1}\right] \mathcal{C}\left[\Lambda_{2}\right] \mathcal{C}\left[\Lambda_{3}\right]\right)\left(\mathcal{C}\left[\bar{\Lambda}_{1}\right] \mathcal{C}\left[\bar{\Lambda}_{2}\right] \mathcal{C}\left[\bar{\Lambda}_{3}\right]\right)
\end{aligned}
$$

Similar calculation gives

$$
\begin{aligned}
& \sum_{1 \in A \subset \Lambda} \sum_{n, m+1 \in D \subset \bar{\Lambda}} \mathcal{K}_{2} \mathcal{C}[A] \mathcal{C}[B] \mathcal{C}[C] \mathcal{C}[D] \\
& =\sum_{\left\langle\Lambda_{1} \Lambda_{2} \Lambda_{3}\right\rangle} \sum_{\left\langle\bar{\Lambda}_{1} \bar{\Lambda}_{2} \bar{\Lambda}_{3}\right\rangle}\left(\mathcal{C}\left[\Lambda_{1}\right] \mathcal{C}\left[\Lambda_{2}\right] \mathcal{C}\left[\Lambda_{3}\right]\right)\left(\mathcal{C}\left[\bar{\Lambda}_{1}\right] \mathcal{C}\left[\bar{\Lambda}_{2}\right] \mathcal{C}\left[\bar{\Lambda}_{3}\right]\right)
\end{aligned}
$$

To finish our proof, let us we consider the last term of relation (4.28)

$$
\begin{aligned}
& \sum_{1 \in A \subset \Lambda} \sum_{n \in D \subset \bar{\Lambda}} \frac{1}{9}\left(s_{A}+s_{B}\right)\left(s_{C}+s_{D}\right) \mathcal{C}[A] \mathcal{C}[B] \mathcal{C}[C] \mathcal{C}[D] \\
= & \sum_{1 \in A \subset \Lambda} \sum_{n \in D \subset \bar{\Lambda}} \frac{1}{9}\left(\sum_{\left\langle A_{1} A_{2}\right\rangle} \mathcal{C}\left[A_{1}\right] \mathcal{C}\left[A_{2}\right] \mathcal{C}[B]+\sum_{\left\langle B_{1} B_{2}\right\rangle} \mathcal{C}[A] \mathcal{C}\left[B_{1}\right] \mathcal{C}\left[B_{2}\right]\right) \\
& \left(\sum_{\left\langle C_{1} C_{2}\right\rangle} \mathcal{C}\left[C_{1}\right] \mathcal{C}\left[C_{2}\right] \mathcal{C}[D]+\sum_{\left\langle D_{1} D_{2}\right\rangle} \mathcal{C}[C] \mathcal{C}\left[D_{1}\right] \mathcal{C}\left[D_{2}\right]\right)
\end{aligned}
$$

with $1 \in A_{1}, n \in D_{1}$. This situation is familiar for us now. There are three configurations for $\mathcal{C}\left[\Lambda_{1}\right] \mathcal{C}\left[\Lambda_{2}\right] \mathcal{C}\left[\Lambda_{3}\right]$ with $1 \in \Lambda_{1}$ and three configurations for $\mathcal{C}\left[\bar{\Lambda}_{1}\right] \mathcal{C}\left[\bar{\Lambda}_{2}\right] \mathcal{C}\left[\bar{\Lambda}_{3}\right]$ with $n \in \bar{\Lambda}_{1}$, thus there are totally nine configuations for the term $\mathcal{C}\left[\Lambda_{1}\right] \mathcal{C}\left[\Lambda_{2}\right] \mathcal{C}\left[\Lambda_{3}\right] \mathcal{C}\left[\bar{\Lambda}_{1}\right] \mathcal{C}\left[\bar{\Lambda}_{2}\right] \mathcal{C}\left[\bar{\Lambda}_{3}\right]$ which leads

$$
\begin{aligned}
& \sum_{1 \in A \subset \Lambda} \sum_{n \in D \subset \bar{\Lambda}} \frac{1}{9}\left(s_{A}+s_{B}\right)\left(s_{C}+s_{D}\right) \mathcal{C}[A] \mathcal{C}[B] \mathcal{C}[C] \mathcal{C}[D] \\
& =\sum_{\left\langle\Lambda_{1} \Lambda_{2} \Lambda_{3}\right\rangle} \sum_{\left\langle\bar{\Lambda}_{1} \bar{\Lambda}_{2} \bar{\Lambda}_{3}\right\rangle}\left(\mathcal{C}\left[\Lambda_{1}\right] \mathcal{C}\left[\Lambda_{2}\right] \mathcal{C}\left[\Lambda_{3}\right]\right)\left(\mathcal{C}\left[\bar{\Lambda}_{1}\right] \mathcal{C}\left[\bar{\Lambda}_{2}\right] \mathcal{C}\left[\bar{\Lambda}_{3}\right]\right)
\end{aligned}
$$

Thus, we have

$$
\begin{aligned}
& \sum_{1, m \in A \subset \Lambda} \sum_{n \in D \subset \bar{\Lambda}} \mathcal{K}_{1} \mathcal{C}[A] \mathcal{C}[B] \mathcal{C}[C] \mathcal{C}[D] \\
& =\sum_{1 \in A \subset \Lambda} \sum_{n,(m+1) \in D \subset \bar{\Lambda}} \mathcal{K}_{2} \mathcal{C}[A] \mathcal{C}[B] \mathcal{C}[C] \mathcal{C}[D] \\
& =\sum_{1 \in A \subset \Lambda} \sum_{n \in D \subset \bar{\Lambda}} \frac{1}{9}\left(s_{A}+s_{B}\right)\left(s_{C}+s_{D}\right) \mathcal{C}[A] \mathcal{C}[B] \mathcal{C}[C] \mathcal{C}[D] \\
& =\sum_{\left\langle\Lambda_{1} \Lambda_{2} \Lambda_{3}\right\rangle} \sum_{\left\langle\bar{\Lambda}_{1} \bar{\Lambda}_{2} \bar{\Lambda}_{3}\right\rangle}\left(\mathcal{C}\left[\Lambda_{1}\right] \mathcal{C}\left[\Lambda_{2}\right] \mathcal{C}\left[\Lambda_{3}\right]\right)\left(\mathcal{C}\left[\bar{\Lambda}_{1}\right] \mathcal{C}\left[\bar{\Lambda}_{2}\right] \mathcal{C}\left[\bar{\Lambda}_{3}\right]\right)
\end{aligned}
$$

This ends the proof of relation (4.28). 
Before ending this section, we would like to point out a by-product in this section. We have shown that the final result of the integration can be expressed as

$$
\begin{aligned}
\mathcal{A}= & \frac{1}{s_{\Lambda}^{3}}\left(\sum_{\langle\Lambda \rightarrow A B\rangle} \sum_{\langle\bar{\Lambda} \rightarrow C D\rangle} \bar{X} \mathcal{C}[A] \mathcal{C}[B] \mathcal{C}[C] \mathcal{C}[D]\right. \\
& -\frac{1}{4} s_{\Lambda} \sum_{\langle\bar{\Lambda} \rightarrow C D\rangle} \sum_{\left\langle\Lambda \rightarrow \Lambda_{1} \Lambda_{2} \Lambda_{3}\right\rangle}(\mathcal{C}[C] \mathcal{C}[D])\left(\mathcal{C}\left[\Lambda_{1}\right] \mathcal{C}\left[\Lambda_{2}\right] \mathcal{C}\left[\Lambda_{3}\right]\right) \\
& -\frac{1}{4} s \sum_{\langle\Lambda \rightarrow A B\rangle} \sum_{\left\langle\bar{\Lambda} \rightarrow \bar{\Lambda}_{1} \bar{\Lambda}_{2} \bar{\Lambda}_{3}\right\rangle}(\mathcal{C}[A] \mathcal{C}[B])\left(\mathcal{C}\left[\bar{\Lambda}_{1}\right] \mathcal{C}\left[\bar{\Lambda}_{2}\right] \mathcal{C}\left[\bar{\Lambda}_{3}\right]\right) \\
& \left.+\frac{1}{4} \sum_{\left\langle\Lambda \rightarrow \Lambda_{1} \Lambda_{2} \Lambda_{3}\right\rangle} \sum_{\left\langle\bar{\Lambda} \rightarrow \bar{\Lambda}_{1} \bar{\Lambda}_{2} \bar{\Lambda}_{3}\right\rangle}\left(\mathcal{C}\left[\Lambda_{1}\right] \mathcal{C}\left[\Lambda_{2}\right] \mathcal{C}\left[\Lambda_{3}\right]\right)\left(\mathcal{C}\left[\bar{\Lambda}_{1}\right] \mathcal{C}\left[\bar{\Lambda}_{2}\right] \mathcal{C}\left[\bar{\Lambda}_{3}\right]\right)\right)
\end{aligned}
$$

where $\bar{X}$ is defined in (4.20). This formulation can be considered as a new kind of integration rule. Now instead of just cubic vertexes like $\langle\Lambda \rightarrow A B\rangle$ and $\langle\bar{\Lambda} \rightarrow C D\rangle$, there are new quartic vertexes like $\left\langle\Lambda \rightarrow \Lambda_{1} \Lambda_{2} \Lambda_{3}\right\rangle$ and $\left\langle\bar{\Lambda} \rightarrow \bar{\Lambda}_{1} \bar{\Lambda}_{2} \bar{\Lambda}_{3}\right\rangle$.

\section{Rule for duplex-double pole}

In this section, we will derive the Feynman rule for duplex-double pole. In [20], the Feynman rule is conjectured to be

$$
\begin{aligned}
\mathcal{R}_{\mathrm{ule}}^{\mathrm{III}}\left[p_{A}, p_{B}, p_{E}, p_{C}, p_{D}\right]= & \frac{\left(2 p_{A} \cdot p_{D}\right)\left(2 p_{B} \cdot p_{C}\right)-\left(2 p_{A} \cdot p_{C}\right)\left(2 p_{B} \cdot p_{D}\right)}{s_{A B}^{2} s_{C D}^{2}} \\
& -\frac{\left(p_{E}^{2}\right)\left(2 p_{A} \cdot p_{D}+2 p_{B} \cdot p_{C}-2 p_{A} \cdot p_{C}-2 p_{B} \cdot p_{D}\right)}{4 s_{A B}^{2} s_{C D}^{2}}
\end{aligned}
$$

In this section, we will derive a different rule for the special case $p_{E}^{2}=0$ for simplicity. The new rule will be different from the one given in (5.1). Since we have restricted ourselves only on the special case, we will not present the equivalent proof of these two rules in this paper.

\subsection{CHY configuration}

Like other two cases, first let us specify the CHY configurations in this section, i.e., all poles are simple poles except two double poles $s_{\Lambda_{1}}$ and $s_{\Lambda_{2}}\left(\Lambda_{1} \bigcap \Lambda_{2}=\emptyset\right)$. Furthermore, to simplify the problem, we have assumed the numerator is one for CHY integrands and the $E=\overline{\Lambda_{1} \bigcup \Lambda_{2}}$ is just a single node $e$. With above specifications, we can derive the following statements:

(1) By the same argument for the case with only one double pole, for the subset $\Lambda_{1}$, there are two points $a, b$ having a line connecting to subset $\bar{\Lambda}_{1}$. Similarly, for the subset $\Lambda_{2}$, there are two points $c, d$ having a line connecting to subset $\bar{\Lambda}_{2}$.

(2) Because $E=\{e\}$, we see immediately that there are four lines [ae], [be], [ce] and $[d e]$. 
(3) For all Feynman diagrams, $\Lambda_{1}$ will split into two simple corners $A$ and $B$ with $a \in A$, $b \in B$, and similarly $\Lambda_{2}$ will split into two simple corners $C$ and $D$ with $c \in C, d \in D$, as can be seen in figure 4 a.

(4) Using (3.5), there is no single pole $\Sigma=\alpha \bigcup \beta$ with true subsets $\alpha \subset \Lambda_{1}, \beta \subset \Lambda_{2}$ by the same argument.

(5) Using (3.5), there is no single pole $\Sigma=\alpha \bigcup E$ with the true subset $\alpha \subset \Lambda_{1}$. The reason is that if $\chi[\alpha]=0$, then $\mathbb{L}[\alpha, E] \leq 1$ since $b, a$ can not belong to same single pole. In other words, we will have $\mathbb{L}[\alpha, E]+\chi[\alpha] \leq 1$. Similarly there is no single pole $\Sigma=\beta \bigcup E$ with the true subset $\beta \subset \Lambda_{2}$.

(6) For the case $\Sigma=\alpha \bigcup \beta \bigcup E$ with true subsets $\alpha \subset \Lambda_{1}, \beta \subset \Lambda_{2}$, we find

$$
\chi[\Sigma]=\chi[\alpha]+\chi[\beta]+\mathbb{L}[\alpha, \beta]+\mathbb{L}[\alpha, E]+\mathbb{L}[\beta, E]-4,
$$

with $\mathbb{L}[\alpha, \beta]=0, \mathbb{L}[\alpha, E]+\chi[\alpha] \leq 1$ and $\mathbb{L}[\beta, E]+\chi[\beta] \leq 1$, we get $\chi[\Sigma]<0$ always, i.e., there is no such a simple pole.

Based on above observations, we see that all maximal compatible combinations must contain $\Lambda_{1}$ and $\Lambda_{2}$. The reason is that there are at most $\left(\left|\Lambda_{1}\right|-2\right)$ compatible combinations from $\Lambda_{1}$ and $\left(\left|\Lambda_{2}\right|-2\right)$ compatible combinations from $\Lambda_{2}$, thus at most we can get $(n-5)$ compatible poles. Therefore one must add two poles $s_{\Lambda_{1}}$ and $s_{\Lambda_{2}}$ to achieve the correct number $(n-3)$ for all poles. In other words, all allowed Feynman diagrams will contain the following cubic vertex where double poles $s_{\Lambda_{1}}$ and $s_{\Lambda_{2}}$ meet with the node $e$. With this picture, the integrated result should be ${ }^{13}$

$$
\begin{aligned}
& I_{\mathrm{CHY}} \rightarrow \sum_{\langle A B C D\rangle} \frac{X}{s_{\Lambda_{1}}^{2} s_{\Lambda_{2}}^{2}} \mathcal{C}[A] \mathcal{C}[B] \mathcal{C}[C] \mathcal{C}[D] \mathcal{C}[E], \\
& a \in A \subset \Lambda_{1}, b \in B=\Lambda_{1} / A, c \in \Lambda_{2} / D, d \in D \subset \Lambda_{2} .
\end{aligned}
$$

Now we need to determine the expression $X$ to get the Feynman rule.

\subsection{Derivation}

Now we derive the rule. Since there are two double poles $s_{\Lambda_{1}}$ and $s_{\Lambda_{2}}$, we need to use the cross-ratio identities twice. In the first step, our choice of the gauge is $\left[a, d, \Lambda_{1}\right]$, then the identity is given by

$$
-1=\sum_{i \in \Lambda_{1} \backslash\{a\}} \sum_{j \in \Lambda_{2} \backslash\{d\}} \frac{s_{i j}}{s_{\Lambda_{1}}} \frac{z_{j d} z_{i a}}{z_{i j} z_{a d}}+\sum_{i \in \Lambda_{1} \backslash\{a\}} \frac{s_{i e}}{s_{\Lambda_{1}}} \frac{z_{e d} z_{i a}}{z_{i e} z_{a d}},
$$

where we have split the sum $j \in \bar{\Lambda}_{1} /\{d\}$ into two parts, as shown in figure $4 \mathrm{~b}$ and figure $4 \mathrm{c}$ respectively. For the first part, the factor $\frac{z_{j d} z_{i a}}{z_{i j} z_{a d}}$ has reduced the double pole $s_{\Lambda_{2}}^{2}$ to simple pole $s_{\Lambda_{2}}$ simultaneously by the numerator $z_{j d}$. Furthermore, it does not create any new simple poles. Firstly, it can not create new simple poles of the form $\alpha \bigcup E$ with $\alpha$ the

\footnotetext{
${ }^{13}$ For single note, since $\mathcal{C}[\{e\}]=1$, one can drop this factor.
} 


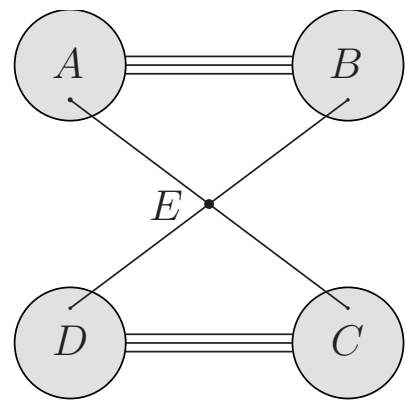

(a) Original integrand

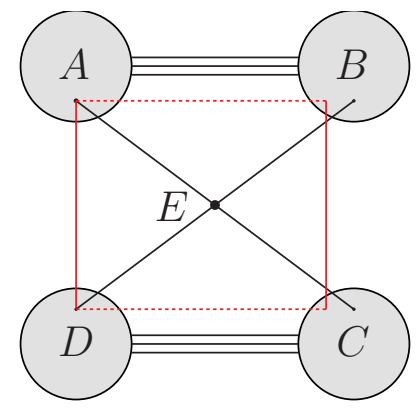

(b) Cross-ratio identity: the $T_{1}$ part

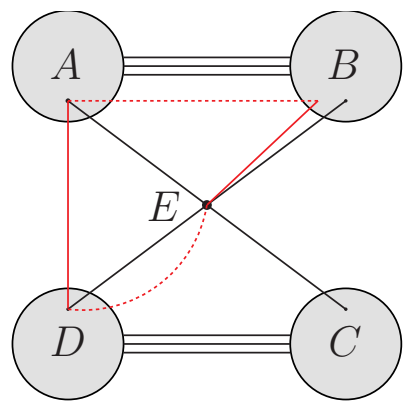

(c) Cross-ratio identity: the $T_{2}$ part

Figure 4. Configuration of the original integrand and the cross-ratio identity of the rule III. The red lines represent terms $\frac{z_{j d} z_{i a}}{z_{i j} z_{a d}}$ and $\frac{z_{e d} z_{i a}}{z_{i e} z_{a d}}$ provided by the cross-ratio identity.

true subsets of either $\Lambda_{1}$ or $\Lambda_{2}$. Secondly, it can not create new simple poles of the form $\alpha \bigcup \beta$ with $\alpha, \beta$ the true subsets of $\Lambda_{1}$ and $\Lambda_{2}$ respectively. Using (3.5), one see that after multiplying the factor $\frac{z_{j d} z_{i a}}{z_{i j} z_{a d}}, L[\alpha, \beta]$ can change from zero to one at most. Similarly, it can not create new simple poles of the form $\alpha \bigcup \beta \bigcup E$ with $\alpha, \beta$ the true subsets of $\Lambda_{1}$ and $\Lambda_{2}$ respectively. For this one, we need to use (5.2) and again $L[\alpha, \beta]$ can change from zero to one at most. Above analysis shows that the first term has been reduced to the case without any higher order poles. Thus the integration rules given in [17-19] can be applied straightforwardly. Putting all considerations together, the first part of (5.4) gives

$$
T_{1}=\sum_{\langle A B C D\rangle} \frac{2 p_{B} \cdot p_{C}}{s_{\Lambda_{1}}} \frac{1}{s_{\Lambda_{1}} s_{\Lambda_{2}}} \mathcal{C}[A] \mathcal{C}[B] \mathcal{C}[C] \mathcal{C}[D] \mathcal{C}[E] .
$$

Now we consider the second part of (5.4). Because of the factor $\frac{z_{e d} z_{i a}}{z_{i e} z_{a d}}$, especially the denominator $z_{i e}$, it is easy to see from (3.5) that now the subset $\alpha \bigcup E$ with $i, b \in \alpha, a \notin \alpha$, $\alpha \subset \Lambda_{1}$ and $\chi[\alpha]=0$ will become a new single pole. This phenomenon will complicate our discussion a lot. Also, for this part, the double pole $s_{\Lambda_{2}}^{2}$ still exists, thus we need to use cross ratio identity again. In fact, if we set $\widetilde{\Lambda}_{1}=\Lambda \bigcup E=\bar{\Lambda}_{2}$ and $\widetilde{\Lambda}_{2}=\Lambda_{2}, I_{\mathrm{org}} \frac{z_{e d} z_{i a}}{z_{i e} z_{a d}}$ is the case with only one double pole $s_{\Lambda_{2}}$ studied in previous section, where $a, e$ are special points in $\widetilde{\Lambda}_{1}$ and $d, c$ are the special points in $\widetilde{\Lambda}_{2}$. Using result (3.21) we get

$$
\frac{\left(2 p_{B} \cdot p_{E}\right)}{s_{\Lambda_{1}}} \sum_{\widetilde{A} \subset \widetilde{\Lambda}_{1}} \sum_{C \subset \Lambda_{2}} \frac{\left(2 p_{\widetilde{A}} \cdot p_{C}\right)}{s_{\Lambda_{2}}^{2}} \mathcal{C}[\widetilde{A}] \mathcal{C}[\widetilde{B}] \mathcal{C}[C] \mathcal{C}[D]
$$

where we have used the fact $\widetilde{\Lambda}_{2}=\Lambda_{2}$. Now coming to the key point: the allowed splitting of $\widetilde{\Lambda}_{1}$ can be divided into two cases. In the first case, $\widetilde{B}=E$ and $\widetilde{A}=\Lambda_{1}$. Furthermore, the $\widetilde{A}$ split into two corners $A, B$ and we have

$$
\mathcal{C}[\widetilde{A}] \mathcal{C}[\widetilde{B}]=\left(\frac{1}{s_{\Lambda_{1}}} \sum_{\langle A B\rangle} \mathcal{C}[A] \mathcal{C}[B]\right) \mathcal{C}[E] .
$$


Putting it back, we get

$$
T_{2 ; 1}=\sum_{\langle A B C D\rangle} \frac{\left(2 p_{B} \cdot p_{E}\right)}{s_{\Lambda_{1}}} \frac{\left(2\left(p_{A}+p_{B}\right) \cdot p_{C}\right)}{s_{\Lambda_{2}}^{2}} \frac{1}{s_{\Lambda_{1}}} \mathcal{C}[A] \mathcal{C}[B] \mathcal{C}[C] \mathcal{C}[D] \mathcal{C}[E]
$$

In the second case, $\widetilde{A}=A$ and $\widetilde{B}=B \bigcup E$. Unlike the first case, we know for sure that $\mathcal{C}[\widetilde{A}]=\left(\frac{1}{s_{\Lambda_{1}}} \sum_{\langle A B\rangle} \mathcal{C}[A] \mathcal{C}[B]\right)$. Here node $e$ can be attached to some propagators in the sub-Feynamn diagrams of subset $B$. We will show that the final result for the second case is

$$
T_{2 ; 2}=\sum_{\langle A B C D\rangle} \frac{\left(2 p_{A} \cdot p_{C}\right) s_{\Lambda_{1}}}{s_{\Lambda_{1}}^{2} s_{\Lambda_{2}}^{2}} \mathcal{C}[A] \mathcal{C}[B] \mathcal{C}[C] \mathcal{C}[D] \mathcal{C}[E] .
$$

Putting (5.5), (5.8) and (5.9) together with proper sign, we get the final result ${ }^{14}$

$$
T_{1}-T_{2 ; 1}-T_{2 ; 2}
$$

which implies that the $X$ in (5.3) is given by

$$
X_{\langle A B C D\rangle}=\left(2 p_{B} \cdot p_{C}\right) s_{\Lambda_{2}}-\left(2 p_{B} \cdot p_{E}\right)\left(2\left(p_{A}+p_{B}\right) \cdot p_{C}\right)-\left(2 p_{A} \cdot p_{C}\right) s_{\Lambda_{1}} .
$$

The Feynman rule (5.3) with $X$ given by (5.11) has been checked numerically with several examples.

Now we explain the result (5.9) for the second splitting of $\widetilde{\Lambda}_{1}=\widetilde{A} \cup \widetilde{B}$ with $\widetilde{A}=A$ and $\widetilde{B}=B \bigcup E$. For a given subset $B$, there are several compatible combinations, i.e., several sub-Feynman diagrams. Let us focus on a particular sub-Feynman diagram $\Gamma$, which is shown in figure $5 \mathrm{a}$. For this $\Gamma$, there is a sequence of single subsets such that $B_{m} \subset B_{m-1} \subset \cdots \subset B_{1} \subset B$ with $B_{m}=\{b\}$. The reason we consider this sequence is that by our previous argument, it is exactly these subsets $B_{t}$, which can combine with the subset $E=\{e\}$ to create a new single pole when the sum $i \in B_{t}$ in the second part of (5.4). In other words, for the second splitting of $\widetilde{\Lambda}_{1}$, the node $e$ will attach exactly to these propagators $B_{t}$ in the Feynman diagram $\Gamma$, as can be seen in figure $5 \mathrm{~b}$. Now we can write down the expression when node $e$ is attached to propagator $B_{t}$ as

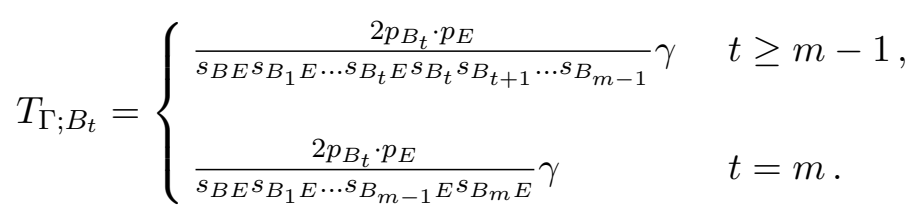

Let us explain the meaning of (5.12). First, to be able to attach $e$ to the propagator $B_{t}$, the sum $i$ in the second part of (5.4) can only be those $i \in B_{t}$, so we get the numerator $2 p_{B_{t}} \cdot p_{E}$. Secondly, along the sequence of propagators, when the node $e$ has been attached, the later propagators will carry corresponding momentum, so we have the propagators $s_{B_{t} E}, s_{B_{t-1} E}, s_{B_{t-2} E}$ until $s_{B E}$. Thirdly, $\gamma=\prod_{t} \gamma_{B_{t}}$ is the other part of propagators, which are not affect by node $e$, as shown in figure 5 . It will be the same for all $T_{\Gamma ; B_{t}}$.

\footnotetext{
${ }^{14}$ The relative minus sign is because for the second part we have inserted another cross ratio identity.
} 


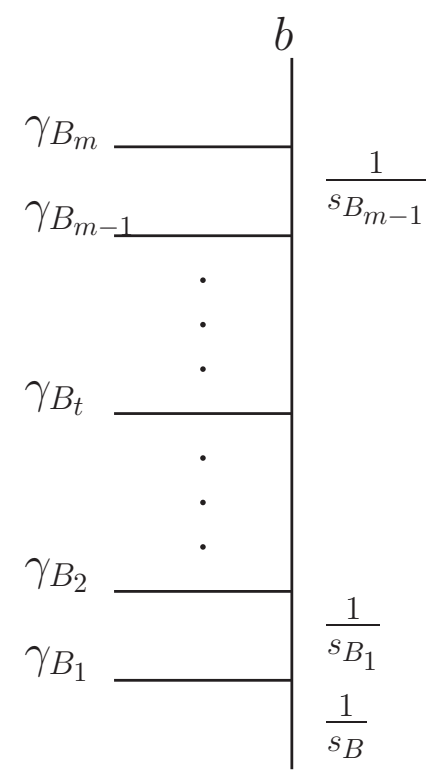

(a) The Feynmann diagram $\Gamma$

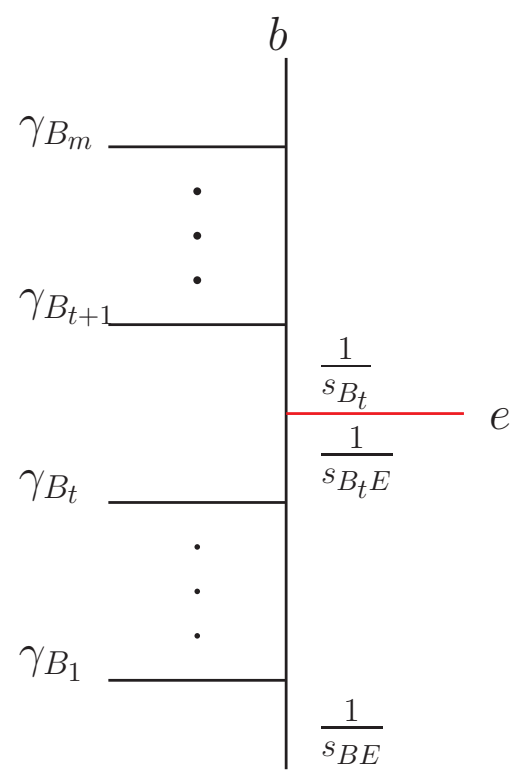

(b) $e$ attaches to the propagator $\frac{1}{s_{B_{t}}}$

Figure 5. The Feynmann diagram $\Gamma$ of the sequence $B_{m} \subset B_{m-1} \subset \cdots \subset B_{1} \subset B$ and the node $e$ attaches to this diagram.

Having obtained the expression, we can carry out the sum. It is easy to see that

$$
\begin{aligned}
T_{\Gamma ; B_{m-1}}+T_{\Gamma ; B_{m}} & =\frac{\gamma}{s_{B E} s_{B_{1} E} \ldots s_{B_{m-2} E} s_{B_{m-1} E}}\left\{\frac{2 p_{B_{m-1}} \cdot p_{E}}{s_{B_{m-1}}}+\frac{2 p_{B_{m}} \cdot p_{E}}{s_{B_{m} E}}\right\} \\
& =\frac{\gamma}{s_{B E} s_{B_{1} E} \ldots s_{B_{m-2} E} s_{B_{m-1} E}} \times \frac{s_{B_{m-1} E}}{s_{B_{m-1}}}=\frac{\gamma}{s_{B E} s_{B_{1} E} \ldots s_{B_{m-2} E} s_{B_{m-1}}},
\end{aligned}
$$

where at the second line we have used the fact $p_{E}^{2}=0$ since $E=\{e\}$ is just a single node. Adding $T_{\Gamma ; B_{m-2}}$, we get

$$
\begin{aligned}
& T_{\Gamma ; B_{m-2}}+T_{\Gamma ; B_{m-1}}+T_{\Gamma ; B_{m}} \\
& =\frac{\gamma}{s_{B E} s_{B_{1} E} \ldots s_{B_{m-2} E}}\left\{\frac{1}{s_{B_{m-1}}}+\frac{2 p_{B_{m-2}} \cdot p_{E}}{s_{B_{m-2}} s_{B_{m-1}}}\right\} \\
& =\frac{\gamma}{s_{B E} s_{B_{1} E} \ldots s_{B_{m-2} E}} \times \frac{s_{B_{m-2} E}}{s_{B_{m-2}} s_{B_{m-1}}}=\frac{\gamma}{s_{B E} s_{B_{1} E} \ldots s_{B_{m-3} E} s_{B_{m-2}} s_{B_{m-1}}} .
\end{aligned}
$$

Now we can see the recursive pattern, which leads to

$$
\sum_{t=0}^{m} T_{\Gamma ; B_{t}}=\frac{\gamma}{s_{B} s_{B_{1}} \ldots s_{B_{m-3}} s_{B_{m-2}} s_{B_{m-1}}} .
$$

This is just the expression of $\Gamma$ itself. This summation can be understood diagrammatically as in figure 6 . This calculation has also shown how the new created poles have been canceled out when summing over all terms. 

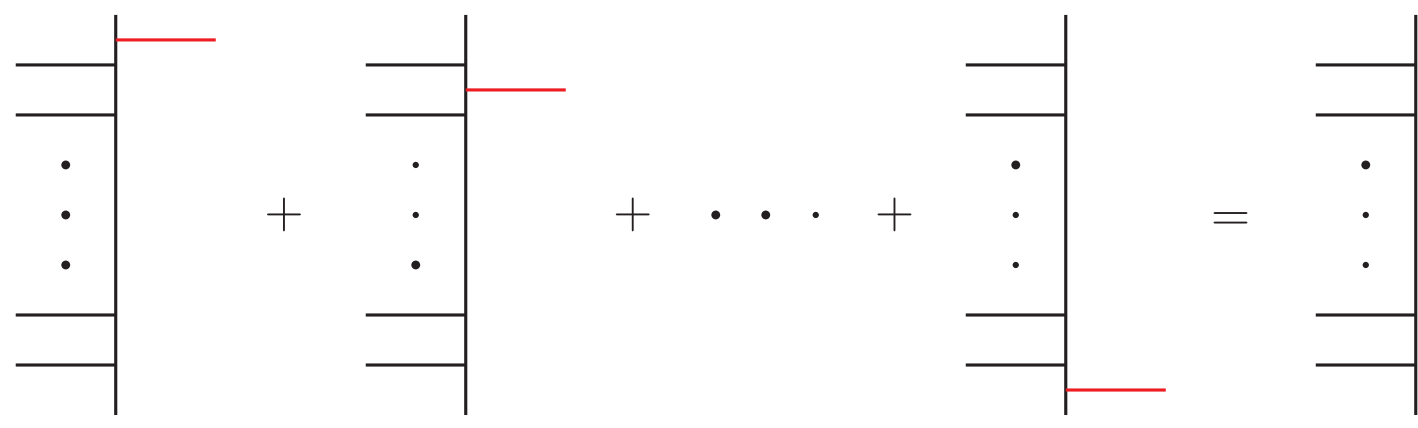

Figure 6. The summation $\sum_{t=0}^{m} T_{\Gamma ; B_{t}}$.

Having above preparations, we are ready to write down the contribution from the second splitting of $\widetilde{\Lambda}_{1}$ as

$$
T_{2 ; 2}=\frac{1}{s_{\Lambda_{1}}} \sum_{\widetilde{\Lambda}_{1}=A \cup \widetilde{B}} \frac{2 p_{A} \cdot p_{C}}{s_{\Lambda_{2}}^{2}} \mathcal{C}[A] \mathcal{C}[C] \mathcal{C}[D] \sum_{\Gamma}\left\{\sum_{t=0}^{m} T_{\Gamma ; B_{t}}\right\},
$$

where the Feynman rule (3.21) has been used. Using (5.15) to above expression, we arrive at the wanted result (5.9).

\section{Conclusion}

In this paper, we use the cross-ratio identity to derive the Feynman rules of higher-order poles in the CHY construction conjectured in [20]. The first rule is valid for CHY integrands containing a double pole, the second rule is valid for integrands containing a triple pole, while the third rule is valid for integrands containing a duplex-double pole. The new expression of rules obtained in this paper depends on the gauge choices of cross-ratio identities, however the final results of integrations after summing over all correct compatible combinations are gauge invariant.

For the first and second rule, we make the comparison of them with those conjectured in [20]. For the second rule, the equivalence between our formula and the conjectured one is non-trivial. We have found that the result of integration can be arranged into a new version of rule which contains quartic vertexes. This is an interesting phenomenon.

For the first two rules, we have performed special gauge choices to ensure that new poles which do not satisfy the desired compatible combination will not emerge. For the third rule however, this idea can not be realized, i.e., one can not avoid the appearing of new poles. Thus we have provided a treatment of new poles for this rule.

In general, searching for integration rules for higher-order poles is not efficient enough for practical computation, since one can not reduce the most general CHY integrands which contain higher order poles into a few configurations. Rules in this paper or rules in [20] only cover some special cases. When encountering new cases, we need new rules. However, since we have proven the conjectured rules through the cross-ratio identities, we can conclude that the cross-ratio identity method is a powerful and universal tool for analytic calculation. 


\section{Acknowledgments}

We would thank Rijun Huang for useful discussions. This work is supported by QiuShi Funding and Chinese NSF funding under contracts No. 11575156, No. 11135006, and No. 11125523 as well as the U.S.A. National Science Foundation under Grant No. NSF PHY-1125915. K.Z also would like to acknowledge the support from Chinese Postdoctoral Administrative Committee. B.F. would like to thank the workshop "Scattering Amplitudes and Beyond" at Kavli Institute for the Theoretical Physics, where the final draft has been edited.

Open Access. This article is distributed under the terms of the Creative Commons Attribution License (CC-BY 4.0), which permits any use, distribution and reproduction in any medium, provided the original author(s) and source are credited.

\section{References}

[1] F. Cachazo, S. He and E.Y. Yuan, Scattering equations and Kawai-Lewellen-Tye orthogonality, Phys. Rev. D 90 (2014) 065001 [arXiv: 1306.6575] [InSPIRE].

[2] F. Cachazo, S. He and E.Y. Yuan, Scattering of Massless Particles in Arbitrary Dimensions, Phys. Rev. Lett. 113 (2014) 171601 [arXiv: 1307.2199] [INSPIRE].

[3] F. Cachazo, S. He and E.Y. Yuan, Scattering of Massless Particles: Scalars, Gluons and Gravitons, JHEP 07 (2014) 033 [arXiv:1309.0885] [INSPIRE].

[4] F. Cachazo, S. He and E.Y. Yuan, Einstein- Yang-Mills Scattering Amplitudes From Scattering Equations, JHEP 01 (2015) 121 [arXiv: 1409.8256] [INSPIRE].

[5] F. Cachazo, S. He and E.Y. Yuan, Scattering Equations and Matrices: From Einstein To Yang-Mills, DBI and NLSM, JHEP 07 (2015) 149 [arXiv:1412.3479] [INSPIRE].

[6] C. Kalousios, Scattering equations, generating functions and all massless five point tree amplitudes, JHEP 05 (2015) 054 [arXiv: 1502.07711] [INSPIRE].

[7] C. Cardona and C. Kalousios, Comments on the evaluation of massless scattering, JHEP 01 (2016) 178 [arXiv: 1509.08908] [INSPIRE].

[8] C. Cardona and C. Kalousios, Elimination and recursions in the scattering equations, Phys. Lett. B 756 (2016) 180 [arXiv:1511.05915] [InSPIRE].

[9] L. Dolan and P. Goddard, General Solution of the Scattering Equations, JHEP 10 (2016) 149 [arXiv: 1511.09441 ] [INSPIRE].

[10] R. Huang, J. Rao, B. Feng and Y.-H. He, An Algebraic Approach to the Scattering Equations, JHEP 12 (2015) 056 [arXiv:1509.04483] [inSPIRE].

[11] M. Søgaard and Y. Zhang, Scattering Equations and Global Duality of Residues, Phys. Rev. D 93 (2016) 105009 [arXiv: 1509.08897] [INSPIRE].

[12] J. Bosma, M. Søgaard and Y. Zhang, The Polynomial Form of the Scattering Equations is an H-Basis, Phys. Rev. D 94 (2016) 041701 [arXiv:1605. 08431] [INSPIRE].

[13] M. Zlotnikov, Polynomial reduction and evaluation of tree- and loop-level CHY amplitudes, JHEP 08 (2016) 143 [arXiv: 1605.08758] [INSPIRE]. 
[14] F. Cachazo and H. Gomez, Computation of Contour Integrals on $\mathcal{M}_{0, n}$, JHEP 04 (2016) 108 [arXiv: 1505.03571] [INSPIRE].

[15] H. Gomez, $\Lambda$ scattering equations, JHEP 06 (2016) 101 [arXiv: 1604.05373] [INSPIRE].

[16] C. Cardona and H. Gomez, Elliptic scattering equations, JHEP 06 (2016) 094 [arXiv: 1605.01446] [INSPIRE].

[17] C. Baadsgaard, N.E.J. Bjerrum-Bohr, J.L. Bourjaily and P.H. Damgaard, Integration Rules for Scattering Equations, JHEP 09 (2015) 129 [arXiv: 1506.06137] [INSPIRE].

[18] C. Baadsgaard, N.E.J. Bjerrum-Bohr, J.L. Bourjaily and P.H. Damgaard, Scattering Equations and Feynman Diagrams, JHEP 09 (2015) 136 [arXiv:1507.00997] [INSPIRE].

[19] C. Baadsgaard, N.E.J. Bjerrum-Bohr, J.L. Bourjaily, P.H. Damgaard and B. Feng, Integration Rules for Loop Scattering Equations, JHEP 11 (2015) 080 [arXiv:1508.03627] [INSPIRE].

[20] R. Huang, B. Feng, M.-x. Luo and C.-J. Zhu, Feynman Rules of Higher-order Poles in CHY Construction, JHEP 06 (2016) 013 [arXiv: 1604.07314] [INSPIRE].

[21] N.E.J. Bjerrum-Bohr, J.L. Bourjaily, P.H. Damgaard and B. Feng, Analytic representations of Yang-Mills amplitudes, Nucl. Phys. B 913 (2016) 964 [arXiv:1605.06501] [INSPIRE].

[22] C. Cardona, B. Feng, H. Gomez and R. Huang, Cross-ratio Identities and Higher-order Poles of CHY-integrand, JHEP 09 (2016) 133 [arXiv:1606.00670] [INSPIRE].

[23] N.E.J. Bjerrum-Bohr, J.L. Bourjaily, P.H. Damgaard and B. Feng, Manifesting Color-Kinematics Duality in the Scattering Equation Formalism, JHEP 09 (2016) 094 [arXiv: 1608.00006] [INSPIRE].

[24] D. Nandan, J. Plefka, O. Schlotterer and C. Wen, Einstein-Yang-Mills from pure Yang-Mills amplitudes, JHEP 10 (2016) 070 [arXiv:1607.05701] [INSPIRE].

[25] R. Huang, Y.-J. Du and B. Feng, Understanding the Cancelation of Double Poles in the Pfaffian of CHY-formulism, arXiv:1702.05840 [INSPIRE].

[26] K. Zhou, Analytic expressions of amplitudes by the cross-ratio identity method, arXiv: 1703.04403 [INSPIRE]. 